\title{
Binding and structure of tetramers in the scaling limit
}

\author{
M. R. Hadizadeh, ${ }^{1, *}$ M. T. Yamashita, ${ }^{1, \dagger}$ Lauro Tomio,,${ }^{1,2, \dagger}$ A. Delfino, ${ }^{2, \S}$ and T. Frederico ${ }^{3, \|}$ \\ ${ }^{1}$ Instituto de Física Teórica, Universidade Estadual Paulista, 01140-070 São Paulo, SP, Brazil \\ ${ }^{2}$ Instituto de Física, Universidade Federal Fluminense, 24210-346 Niterói, RJ, Brazil \\ ${ }^{3}$ Instituto Tecnológico de Aeronáutica, DCTA, 12228-900 São José dos Campos, SP, Brazil
}

(Received 23 October 2011; published 8 February 2012)

\begin{abstract}
The momentum-space structure of the Faddeev-Yakubovsky (FY) components of weakly bound tetramers is investigated at the unitary limit using a renormalized zero-range two-body interaction. The results, obtained by considering a given trimer level with binding energy $B_{3}$, provide further support to a universal scaling function relating the binding energies of two successive tetramer states. The correlated scaling between the tetramer energies comes from the sensitivity of the four-boson system to a short-range four-body scale. Each excited $N$ th tetramer energy $B_{4}^{(N)}$ moves as the short-range four-body scale changes, while the trimer properties are kept fixed, with the next excited tetramer $B_{4}^{(N+1)}$ emerging from the atom-trimer threshold for a universal ratio $B_{4}^{(N)} / B_{3}=B_{4}^{(N)} / B_{4}^{(N+1)} \simeq 4$.6, which does not depend on $N$. We show that both channels of the FY decomposition [atom-trimer ( $K$ type) and dimer-dimer $(H$ type)] present high-momentum tails that reflect the short-range four-body scale. We also found that the $H$ channel is favored over the $K$ channel at low momentum, when the four-body momentum scale largely overcomes the three-body scale.
\end{abstract}

DOI: 10.1103/PhysRevA.85.023610

PACS number(s): 67.85.-d, 03.65.Ge, 05.10.Cc, 21.45.-v

\section{INTRODUCTION}

A limit cycle in physics [1] refers to a model-independent way to look for a manifestation of a hidden scale, one in which a correlation between physical quantities is geometrically rescaled and replicates itself. Limit cycles are shown to exist even in simple quantum three-boson systems [2-4], manifested for large two-body scattering lengths as well as for short-range interactions. They follow the 1970 Efimov key prediction [5] of geometrically separated, weakly bound, three-boson states near the scattering threshold, confirmed by recent experiments in cold-atom traps [6-8]. For a more recent exposition on the experimental status, see Ref. [9]. The addition of one more particle to the quantum three-body system has long challenged this picture [10-13]. Recent theoretical works [14-21] motivated by cold-atom experiments [22-24] have revived this issue. The actual relevance in identifying simple universal relations in few-body binding laws, with the corresponding experimental possibilities, have been discussed by Modugno [25] in a recent perspective article on this matter.

In Ref. [26] we reported a different dimension of this problem by establishing a universal correlation among the binding energies of two successive tetramer states between two Efimov trimers through precise numerical calculations within a zero-range interaction at the unitary limit (zero two-body binding). It was shown that such tetramer states are related to an unsuspected limit cycle not constrained by Efimov physics or by three-body properties. Tetramers hitting the atom-trimer threshold, leading to resonant recombination losses in this channel, are signatures of a new limit cycle. Furthermore, other four-boson observables close to the unitary limit can exhibit correlations not constrained by the low-energy, twoand three-boson properties. Recombination rates, atom-trimer

\footnotetext{
*hadizade@ift.unesp.br

†yamashita@ift.unesp.br

†tomio@ift.unesp.br

$\S$ delfino@if.uff.br

"tobias@ita.br
}

or dimer-dimer scattering lengths, can move near the Feshbach resonance (FR) independently of the two- and three-body properties. The appearance of few-body forces in the open channel, due to the coupling with the closed channel, can drive the four-body physics [15]. Indeed, there is recent experimental evidence, reported by Nakajima et al. [27], of a three-body force acting in the open channel near the FR in a three-component mixture of a ${ }^{6} \mathrm{Li}$ cold gas.

An appropriate way to directly probe the universal properties of a few-boson system at low energy near the unitary limit is to present results in terms of scaling functions correlating pairs of observables, within a renormalized zero-range two-body interaction. In this way, no effect can be claimed to originate from a particular form of the short-range interaction between the particles, as evidenced by the three-body universal scaling function derived in Ref. [2]. As shown in this case, the regularization parameter in the renormalized zero-range approach can be directly associated with a three-boson physical property, in addition to a two-body observable, such as the scattering length. The concept of scaling functions, which is used to evidence universal properties of three-boson systems close to the unitary limit, is extended to four-boson systems in Ref. [26], as well as in the present work. Within this context, we study the sensitivity of tetramer properties to a four-boson scale by using the Faddeev-Yakubovsky (FY) formalism [28,29] with zero-range two-body interactions. Therefore, independently of the choice of the three-body scale that fixes the trimer properties [15], a four-body regularization parameter (four-body scale) is introduced in the FY formalism. The new short-range scale indeed has effects on four-boson physics, which appears as universal correlations between four-boson low-energy $s$-wave observables not constrained by the trimer properties. The four-body quantities change as long as the new scale varies.

Previous conclusions for the nonexistence of a proper four-body scale rely on a strong suppression of the short-range physics, beyond that already accounted for in the three-boson system. Indeed, by estimating the trace of the kernel of the fourbody equation in momentum space, Amado and Greenwood 
[10] showed that there is no infrared divergence, which led them to conclude against the existence of the Efimov effect in the case of four or more particles. However, the momentum integrals should also implicitly have an ultraviolet cutoff (the four-body one) to regulate them. Note that according to Weinberg's eigenvalue criterion [30], the divergence of the trace is a necessary condition for an infinite number of bound states. However, in the kernel of the FY equation, this criterion can only by applied when considering the trimer in the ground state. More explicitly, in case of ultraviolet divergence of the four-body kernel, by moving a four-body scale in relation to the three-body one, an infinite number of tetramer states emerges from the threshold, but the number is restricted by the energy interval between trimers (when the reference trimer is an excited one), such that it can be infinite only in case the given trimer is the ground state.

It is also instructive to recall other pioneering four-body calculations that have verified the possibility of Efimov-like behavior in four-body systems, such as the works of Kröger and Perne [11] and Naus and Tjon [13]. Conclusions drawn within the nuclear physics context are obviously limited, in view of the strong nuclear repulsion of the potential core, such that the possible observation of a four-body scale, independent from the three- and two-body ones, is suppressed. It explains why the ${ }^{4} \mathrm{He}$ and the triton binding energies are strongly correlated with a fixed slope, as verified by Tjon [31]. In our understanding of the four-body problem, two parameters are not enough (which determines trimer properties) to describe the fourboson system. Our results imply that the correlation between the tetramer and trimer binding energies forms a family of Tjon lines with slopes depending on the new four-boson scale. To exemplify that, we have shown a four-boson universal correlation among the energies of two successive tetramers appearing between two consecutive Efimov states [26]. The correlation exhibits a dependence on a new scale not fixed only by the trimer properties. In order to directly address this novel universal behavior, we have performed a number of calculations of tetramer properties within a zero-range model to show how the dependence on the new short-range scale is evidenced through their structure in momentum space.

The tetramer energies can be presented in a scaling plot where the behavior of a four-body scale can be easily verified in comparison with other relevant few-body scales [26]. The existence of a short-range four-body scale, which is independent of the two- and three-body scales, is expressed in terms of scaling functions or correlation between observables. Once the short-range parameters are eliminated in favor of physical quantities, the renormalized results appear as correlations between the four-body observables with fixed dimer and trimer properties.

In the present work we provide details for the approach that lead us to report [26] a universal correlation between the energies of two successive tetramers. As is thoroughly presented in the next sections, we establish by our numerical investigations that a new short-range parameter is necessary to fix the properties of the four-boson system in the unitary limit. Our conclusions are supported by precise numerical solutions of the four-boson FY integral equations in momentum space for a zero-range two-body interaction [15], as well as by an analysis of the four-body wave function.
The paper is organized as follows. In Sec. II we present the FY formalism within a renormalized zero-range interaction. The relevant scales are introduced in the formalism through a subtractive renormalization approach. In Sec. III the scaling functions for the binding energies of trimers and tetramers are discussed, following Refs. [2] and [26]. Numerical results close to the unitary limit are also reported in this section. In Sec. IV we present our main results for the structure of universal tetramers. We include in this section an extended analysis of the low- and high-momentum structures of the atom-trimer $[(\mathrm{A}+\mathrm{T})$ or $K$-type $]$ and dimer-dimer $[(\mathrm{D}+\mathrm{D})$ or $H$-type] components of the FY decomposition to pin down where the manifestation of the short-range four-body scale is more evident. The behavior of the four-body FY components of the wave function is presented in this section. Finally, in Sec. $\mathrm{V}$ we present our conclusions with perspectives in relation to possible experimental observations. Three appendices supply further details regarding our approach. In Appendixes A and B we have details on the four-body formalism and total wave function in momentum space. In Appendix $\mathrm{C}$ we discuss the stability and convergence of our numerical approach, and give some details on the Lanczos-type procedure for solution of the coupled FY equations.

\section{FADDEEV-YAKUBOVSKY FORMALISM}

The treatment of four-body problems is quite well known in quantum-scattering theory, following the original Faddeev formulation of the three-body systems [28], later on extended to $N$-particle scattering by Yakubovsky [29]. The actually known Faddeev-Yakubovsky formalism has been considered by several authors in their effort to solve different aspects of four-body systems in nuclear and atomic physics. In addition to the works on four-body systems already cited in the Introduction, we can also mention several other reference works concerning details of the bound-state four-body FY formalism, such as Refs. [32-41] and references therein. Alternatively, in the solution of four-body systems with separable interactions, several authors (exemplified by Refs. [42-45]) have applied the Alt-Grassberger-Sandhas formalism [46], in which the $t$-matrix components are used instead of the wave functions.

Here we describe the FY formalism for bound states of four identical bosons by considering general separable twobody interactions. Within our approach, the form factors of the separable potential are conveniently replaced by pointlike interactions within a renormalized zero-range model where the three- and four-body scales are introduced.

\section{A. Bound-state equations and notation}

In a four-body (4B) system (with particles $i, j, k$, and $l$ ) there are 18 different coordinate systems, each one associated with a specific two-body partition (see Appendices A and B). Basically, by considering the arrangements of the corresponding two- and three-body subsystems, two different partitions are possible: $K$ type $(\mathrm{A}+\mathrm{T})$, where a single particle is bound to a three-body subsystem, and $H$ type $(\mathrm{D}+\mathrm{D})$, where two dimers are bound. Clearly, as we know from the Faddeev formalism, each three-body subsystem also has three atom-dimer partitions.

We present below the FY formalism with our notation, which is used along the next sections. The bound state of four 
particles which interact via pairwise forces $V_{i j}$ is given by the Schrödinger equation,

$$
|\Psi\rangle=G_{0} \sum_{i<j} V_{i j}|\Psi\rangle=\sum_{i<j}\left|\psi_{i j}\right\rangle,
$$

where $\left|\psi_{i j}\right\rangle=G_{0} V_{i j}|\Psi\rangle, G_{0}=\left(E-H_{0}\right)^{-1}$ is the free fourbody resolvent and $H_{0}$ stands for the free Hamiltonian. The above components satisfy

$$
\begin{aligned}
\left|\psi_{i j}\right\rangle & =G_{0} t_{i j} \sum_{k l \neq i j}\left|\psi_{k l}\right\rangle \\
& =G_{0} t_{i j}\left(\left|\psi_{i k}\right\rangle+\left|\psi_{i l}\right\rangle+\left|\psi_{j k}\right\rangle+\left|\psi_{j l}\right\rangle+\left|\psi_{k l}\right\rangle\right),
\end{aligned}
$$

where the two-body transition operator is $t_{i j}$. The FY decomposition of $\left|\psi_{i j}\right\rangle$ reads

$$
\begin{aligned}
\left|K_{i j, k}^{l}\right\rangle & =G_{0} t_{i j}\left(\left|\psi_{i k}\right\rangle+\left|\psi_{j k}\right\rangle\right), \\
\left|K_{i j, l}^{k}\right\rangle & =G_{0} t_{i j}\left(\left|\psi_{i l}\right\rangle+\left|\psi_{j l}\right\rangle\right), \\
\left|H_{i j, k l}\right\rangle & =G_{0} t_{i j}\left|\psi_{k l}\right\rangle,
\end{aligned}
$$

where $\left|K_{i j, k}^{l}\right\rangle$ and $\left|H_{i j, k l}\right\rangle$ correspond, respectively, to $K$-type and $H$-type partitions. The Faddeev components of the wave function

$$
\left|\psi_{i j}\right\rangle=\left|K_{i j, k}^{l}\right\rangle+\left|K_{i j, l}^{k}\right\rangle+\left|H_{i j, k l}\right\rangle
$$

are reconstructed through FY components.

Every $\left|\psi_{i j}\right\rangle$ component contains two $K$-type and one $H$-type configurations. Therefore, the total wave function $|\Psi\rangle$ contains twelve different $K$-type chains and six $H$-type chains, leading to 18 independent FY components. By considering identical bosons, the four-body wave function $|\Psi\rangle$ has to be totally symmetric. As a consequence, all $12 K$-type components are identical in their functional form with particles permuted. The same is true for the six $H$-type components. Thus it is sufficient to consider only two independent FY components corresponding to the $K$ - and $H$-type partitions, $|K\rangle \equiv\left|K_{i j, k}^{l}\right\rangle$ and $|H\rangle \equiv\left|H_{i j, k l}\right\rangle$. The 18 coupled FY equations, for identical bosons, shrink to two coupled homogeneous equations,

$$
\begin{aligned}
\left|K_{i j, k}^{l}\right\rangle & =G_{0} t_{i j} P\left[\left(1+P_{k l}\right)\left|K_{i j, k}^{l}\right\rangle+\left|H_{i j, k l}\right\rangle\right], \\
\left|H_{i j, k l}\right\rangle & =G_{0} t_{i j} \tilde{P}\left[\left(1+P_{k l}\right)\left|K_{i j, k}^{l}\right\rangle+\left|H_{i j, k l}\right\rangle\right],
\end{aligned}
$$

where $P_{k l}$ is the permutation operator for the pair $(k l)$, with $P$ and $\tilde{P}$ defined by

$$
P=\left(P_{i j}+P_{i k}\right) P_{j k} \quad \text { and } \quad \tilde{P}=P_{i k} P_{j l} .
$$

The symmetry property of $|K\rangle$ under exchange of particles $i$ and $j$, and $|H\rangle$ under separate exchanges of particles $i, j$ and $k, l$, guarantee that the full wave function

$$
\begin{aligned}
|\Psi\rangle= & \left(1+P+P_{k l} P+\tilde{P}\right)\left[\left(1+P_{k l}\right)\left|K_{i j, k}^{l}\right\rangle+\left|H_{i j, k l}\right\rangle\right] \\
= & {\left[1+(1+P) P_{k l}\right](1+P)\left|K_{i j, k}^{l}\right\rangle } \\
& +(1+P)(1+\tilde{P})\left|H_{i j, k l}\right\rangle
\end{aligned}
$$

is totally symmetric. In order to get insight on how the shortrange four-body scale is reflected in the bosonic wave function for a zero-range force, we will analyze the momentum structure of the $K$ and $H$ components. Following this strategy we will be able to map where the new scale is more relevant to build the tetramer wave function. For that purpose, we still simplify
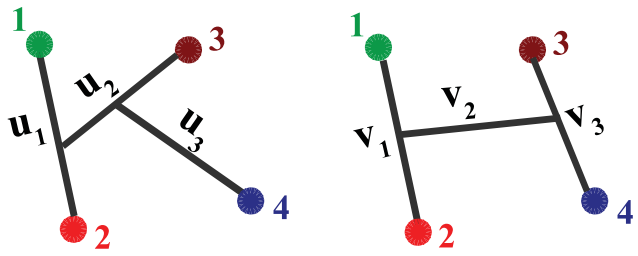

FIG. 1. (Color online) Definition of the four-body Jacobi momenta corresponding to the $K$ - and $H$-type fragmentations.

the problem using $s$-wave one-term separable potentials. This class contains, in particular, the contact interaction.

\section{B. One-term separable potential with $s$-wave projection}

In this section, to simplify the notation we use the particle labels 1,2,3, and 4. Then we rewrite Eq. (5) as

$$
\begin{aligned}
& |K\rangle=G_{0} t_{12} P\left[\left(1+P_{34}\right)|K\rangle+|H\rangle\right], \\
& |H\rangle=G_{0} t_{12} \tilde{P}\left[\left(1+P_{34}\right)|K\rangle+|H\rangle\right] .
\end{aligned}
$$

In order to solve the coupled Eqs. (8) in momentum space, one should project these equations into standard sets of Jacobi momenta, corresponding to both $K$-type $\left(\left|\mathbf{u}_{1} \mathbf{u}_{2} \mathbf{u}_{3}\right\rangle\right)$ and $H$ type $\left(\left|\mathbf{v}_{1} \mathbf{v}_{2} \mathbf{v}_{3}\right\rangle\right)$ partitions, as represented in Fig. 1.

The standard Jacobi momenta for the $4 \mathrm{~B}$ system can be defined in terms of the single-particle momentum variables $\mathbf{k}_{i=1,2,3,4}$, in two possible configurations ( $K$ and $H$ type), as shown in Fig. 1:

$$
\begin{aligned}
& \mathbf{u}_{1}=(1 / 2)\left(\mathbf{k}_{1}-\mathbf{k}_{2}\right), \\
& \mathbf{u}_{2}=(1 / 3)\left[2 \mathbf{k}_{3}-\left(\mathbf{k}_{1}+\mathbf{k}_{2}\right)\right], \\
& \mathbf{u}_{3}=(1 / 4)\left[3 \mathbf{k}_{4}-\left(\mathbf{k}_{1}+\mathbf{k}_{2}+\mathbf{k}_{3}\right)\right], \\
& \mathbf{v}_{1}=(1 / 2)\left(\mathbf{k}_{1}-\mathbf{k}_{2}\right), \\
& \mathbf{v}_{2}=(1 / 2)\left[\left(\mathbf{k}_{1}+\mathbf{k}_{2}\right)-\left(\mathbf{k}_{3}+\mathbf{k}_{4}\right)\right], \\
& \mathbf{v}_{3}=(1 / 2)\left(\mathbf{k}_{3}-\mathbf{k}_{4}\right),
\end{aligned}
$$

where we assume identical particles, with mass $m=1$. Since we are interested in the $s$-wave channel contribution, we introduce the partial-wave representation of the four-body projection operators corresponding to each Jacobi momenta set as

$$
|u\rangle \equiv\left|u_{1} u_{2} u_{3}\right\rangle, \quad|v\rangle \equiv\left|v_{1} v_{2} v_{3}\right\rangle .
$$

Within the $s$-wave projection, we consider the following completeness relation for both basis sets:

$$
\int D^{3} U|U\rangle\langle U|=1,
$$

where $U$ indicates each one of $u$ and $v$ sets, with $D^{3} U \equiv$ $U_{1}^{2} d U_{1} U_{2}^{2} d U_{2} U_{3}^{2} d U_{3}$. Clearly, the projection operators $|u\rangle$ and $|v\rangle$ are adequate to expand the $|K\rangle$ and $|H\rangle$ components, respectively. Consequently, the projection of the coupled equations (8) is given by

$$
\begin{aligned}
& \langle u \mid K\rangle=\left\langle u\left|G_{0} t P\left(1+P_{34}\right)\right| K\right\rangle+\left\langle u\left|G_{0} t P\right| H\right\rangle, \\
& \langle v \mid H\rangle=\left\langle v\left|G_{0} t \tilde{P}\left(1+P_{34}\right)\right| K\right\rangle+\left\langle v\left|G_{0} t \tilde{P}\right| H\right\rangle .
\end{aligned}
$$

In the following, the units are such that $\hbar=1$ and $m=1$.

By considering a one-term separable two-body potential operator $V=\lambda|\chi\rangle\langle\chi|$, the $s$-wave two-body $t$-matrix elements 
can be written, in the $K$ and $H$ representations, by

$$
\left\langle U|t| U^{\prime}\right\rangle=4 \pi \frac{\delta\left(U_{2}^{\prime}-U_{2}\right)}{U_{2}^{2}} \frac{\delta\left(U_{3}^{\prime}-U_{3}\right)}{U_{3}^{2}} \chi\left(U_{1}\right) \chi\left(U_{1}^{\prime}\right) \tau\left(\epsilon_{U}\right),
$$

where the corresponding $K$-type and $H$-type Jacobi sets, $\left\{u_{1}, u_{2}, u_{3}\right\}$ and $\left\{v_{1}, v_{2}, v_{3}\right\}$, are represented by $\left\{U_{1}, U_{2}, U_{3}\right\}$. In the above, $\tau\left(\varepsilon_{U}\right)$ is the reduced scattering amplitude, given by

$$
\tau\left(\mathcal{E}_{U}\right) \equiv\left[\frac{1}{\lambda}-\int d^{3} p \frac{\chi^{2}(p)}{\mathcal{E}_{U}-p^{2}+\mathrm{i} \delta}\right]^{-1},
$$

where, for the $K$-type and $H$-type configurations, respectively, we have

$$
\mathcal{E}_{u} \equiv E-\frac{3 u_{2}^{2}}{4}-\frac{2 u_{3}^{2}}{3} \quad \text { and } \quad \mathcal{E}_{v} \equiv E-\frac{v_{2}^{2}}{2}-v_{3}^{2} .
$$

Note that $\mathcal{E}_{u}$ and $\mathcal{E}_{v}$ have different expressions in the $K$ and $H$ representations but are identical when written in terms of the single-particle momentum coordinates $\mathbf{k}_{i}$.

The final expressions for the FY components, derived in Appendix A through Eqs. (A1)-(A15), are

$$
\begin{aligned}
K\left(u_{1}, u_{2}, u_{3}\right)= & 4 \pi G_{0}\left(u_{1}, u_{2}, u_{3}\right) \chi\left(u_{1}\right) \tau\left(\mathcal{E}_{u}\right) \int_{0}^{\infty} d u_{2}^{\prime} u_{2}^{\prime 2} \int_{-1}^{1} d x \chi\left(\Pi_{1}\left(u_{2}, u_{2}^{\prime}, x\right)\right)\left[K\left(\Pi_{1}\left(u_{2}^{\prime}, u_{2}, x\right), u_{2}^{\prime}, u_{3}\right)\right. \\
& \left.+\frac{1}{2} \int_{-1}^{1} d x^{\prime}\left\{K\left(\Pi_{1}\left(u_{2}^{\prime}, u_{2}, x\right), \Pi_{2}\left(u_{2}^{\prime}, u_{3}, x^{\prime}\right), \Pi_{3}\left(u_{2}^{\prime}, u_{3}, x^{\prime}\right)\right)+H\left(\Pi_{1}\left(u_{2}^{\prime}, u_{2}, x\right), \Pi_{4}\left(u_{2}^{\prime}, u_{3}, x^{\prime}\right), \Pi_{5}\left(u_{2}^{\prime}, u_{3}, x^{\prime}\right)\right)\right\}\right], \\
H\left(v_{1}, v_{2}, v_{3}\right)= & \left.4 \pi G_{0}\left(v_{1}, v_{2}, v_{3}\right) \chi\left(v_{1}\right) \tau\left(\mathcal{E}_{v}\right) \int_{0}^{\infty} d v_{3}^{\prime} v_{3}^{\prime 2} \chi\left(v_{3}^{\prime}\right)\left[\int_{-1}^{1} d x K\left(v_{3}, \Pi_{6}\left(v_{2}, v_{3}^{\prime}, x\right), \Pi_{7}\left(v_{2}, v_{3}^{\prime}, x\right)\right)+H\left(v_{3}, v_{2}, v_{3}^{\prime}\right)\right], \quad(17)\right]
\end{aligned}
$$

where the functions $\Pi_{J=1,2 \ldots 7}$ are defined by Eqs. (A5), (A10), (A11), (A13), (A14), (A16), and (A17). Equations (17) can be rewritten within new definitions for the FY components, such as

$$
\begin{aligned}
K\left(u_{1}, u_{2}, u_{3}\right) & \equiv G_{0}\left(u_{1}, u_{2}, u_{3}\right) \chi\left(u_{1}\right) \mathcal{K}\left(u_{2}, u_{3}\right), \\
H\left(v_{1}, v_{2}, v_{3}\right) & \equiv G_{0}\left(v_{1}, v_{2}, v_{3}\right) \chi\left(v_{1}\right) \mathcal{H}\left(v_{2}, v_{3}\right)
\end{aligned}
$$

Furthermore, by considering a zero-range potential, with $\chi=1$, the above coupled equations (17) are reduced to the following, where the need for regularization in the momentum integrals is explicit:

$$
\begin{aligned}
\mathcal{K}\left(u_{2}, u_{3}\right)= & 4 \pi \tau\left(\mathcal{E}_{u}\right) \int_{0}^{\infty} d u_{2}^{\prime} u_{2}^{\prime 2} \int_{-1}^{1} d x\left[G_{0}\left(\Pi_{1}\left(u_{2}^{\prime}, u_{2}, x\right), u_{2}^{\prime}, u_{3}\right) \mathcal{K}\left(u_{2}^{\prime}, u_{3}\right)\right. \\
& +\frac{1}{2} \int_{-1}^{1} d x^{\prime}\left\{G_{0}\left(\Pi_{1}\left(u_{2}^{\prime}, u_{2}, x\right), \Pi_{2}\left(u_{2}^{\prime}, u_{3}, x^{\prime}\right), \Pi_{3}\left(u_{2}^{\prime}, u_{3}, x^{\prime}\right)\right) \mathcal{K}\left(\Pi_{2}\left(u_{2}^{\prime}, u_{3}, x^{\prime}\right), \Pi_{3}\left(u_{2}^{\prime}, u_{3}, x^{\prime}\right)\right)\right. \\
& \left.\left.+G_{0}\left(\Pi_{1}\left(u_{2}^{\prime}, u_{2}, x\right), \Pi_{4}\left(u_{2}^{\prime}, u_{3}, x^{\prime}\right), \Pi_{5}\left(u_{2}^{\prime}, u_{3}, x^{\prime}\right)\right) \mathcal{H}\left(\Pi_{4}\left(u_{2}^{\prime}, u_{3}, x^{\prime}\right), \Pi_{5}\left(u_{2}^{\prime}, u_{3}, x^{\prime}\right)\right)\right\}\right] \\
\mathcal{H}\left(v_{2}, v_{3}\right)= & 4 \pi \tau\left(\mathcal{E}_{v}\right) \int_{0}^{\infty} d v_{3}^{\prime} v_{3}^{\prime 2}\left[\int_{-1}^{1} d x G_{0}\left(v_{3}, \Pi_{6}\left(v_{2}, v_{3}^{\prime}, x\right), \Pi_{7}\left(v_{2}, v_{3}^{\prime}, x\right)\right) \mathcal{K}\left(\Pi_{6}\left(v_{2}, v_{3}^{\prime}, x\right), \Pi_{7}\left(v_{2}, v_{3}^{\prime}, x\right)\right)\right. \\
& \left.+G_{0}\left(v_{3}, v_{2}, v_{3}^{\prime}\right) \mathcal{H}\left(v_{2}, v_{3}^{\prime}\right)\right]
\end{aligned}
$$

Following an appropriate renormalization scheme for zerorange interactions, with the regularization parameters directly associated with observables [2], in the regularization of the above coupled equations we introduce the short-range threeand four-body scales that we discuss in the following topic of this section.

\section{Four-boson zero-range bound-state model}

In order to aid the understanding of the zero-range model in the four-boson formalism, we briefly review the main steps to deal with two- and three-boson equations when we have two-body zero-range interactions. The momentum-space representation of a zero-range interaction $V(\mathbf{r})=(2 \pi)^{3} \lambda \delta(\mathbf{r})$ is characterized by the two-body coupling constant $\lambda$ and a separable potential with constant form factor $(\langle\mathbf{p} \mid \chi\rangle=1)$. The matrix element of the two-body transition operator, $\left\langle p^{\prime}|t(\mathcal{E})| p\right\rangle=\tau(\mathcal{E})$, is obtained by analytical integration of Eq. (15). The coupling constant $\lambda$ is fixed by one physical input, e.g., the position of the two-body pole at $E_{2}$ :

$$
\lambda^{-1}=\int d^{3} p \frac{1}{E_{2}-p^{2}}=-\int d^{3} p \frac{1}{\left|E_{2}\right|+p^{2}} .
$$

We consider bound or virtual two-body energies, when $E_{2}=$ $-\left|E_{2}\right|$-bound when the pole is in the upper part of the 
complex imaginary axis of the momentum plane $\left(\mathrm{i} \kappa_{B}\right)$, with corresponding energy in the first Riemann sheet $\left(E_{2}=-\kappa_{B}^{2}\right)$, and virtual when the pole is in the lower part of the complex imaginary axis $\left(-\mathrm{i} \kappa_{v}\right)$, with the energy in the second Riemann sheet $\left(E_{2}=-\kappa_{v}^{2}\right)$. As we are going to consider one case or the other, both $\kappa_{B}$ and $\kappa_{V}$ are labeled as $\sqrt{B_{2}}$. Therefore the corresponding scattering lengths are given by $a= \pm 1 / \sqrt{B_{2}}$ ( + for bound, and - for virtual), with the renormalized two-body $t$ matrix given by

$$
\tau(\mathcal{E})=\frac{1 /\left(2 \pi^{2}\right)}{\sqrt{\left|E_{2}\right|}-\sqrt{-\mathcal{E}}}=\frac{1 /\left(2 \pi^{2}\right)}{ \pm 1 / a-\sqrt{|\mathcal{E}|}} .
$$

The above procedure is enough to render finite the scattering amplitude, providing cancellation of the linear divergence of the momentum integral.

\section{Subtractive regularization technique}

The subtraction technique used to define the two-body scattering amplitude can be generalized to three-boson systems. The need for a new parameter beyond $a$ is demanded by the Thomas collapse [47] of the three-particle ground state, when the two-body interaction range $r_{0}$ goes to zero. Such collapse is also associated with the Efimov phenomenon by observing that in both cases $|a| / r_{0} \rightarrow \infty$ [48]. The Thomas collapse is avoided at the expense of regularizing the kernel of the threebody equation, which is done by introducing a subtraction at an energy scale $-\mu_{3}^{2}$. Together with the two-body scattering length, this procedure determines the low-energy three-boson properties. The regularization of the momentum integration is done by the subtracted form $G_{0}(E)-G_{0}\left(-\mu_{3}^{2}\right)$ in substitution to the free three-body Green's function $G_{0}(E)$. The regularizing energy parameter $\mu_{3}^{2}$ is also called a "three-body scale" in view of its direct association to a three-body physical observable (scale) in the renormalization procedure. See, e.g., Ref. [57] for a review on the physical scales and the subtractive regularization technique.

By adding a fourth particle to the three-body system, within the same zero-range two-body interaction, another regularization is required in the corresponding formalism due to new terms in the coupled integral equations, not directly identified with the three-body kernel. This regularization is followed in a similar way as for the three-boson case-by introducing one more regularizing parameter ("four-body scale"), $\mu_{4}^{2}$, in the FY formalism. This new scaling parameter appears in the integrands associated with the presence of the fourth particle in order to allow the complete regularization of all the momentum integrals. Among the 18 FY components, only the first three (i.e., $i j k+l, j k i+l$, and $k i j+l$ ) will fully describe the three-body (3B) system $(i j k)$, where the $3 \mathrm{~B}$ scaling parameter $\mu_{3}^{2}$ enters in the subtracted form of the free Green's function. The 4B scaling parameter $\mu_{4}^{2}$ enters in the subtracted form of the Green's function that is present in the remaining 15 components. Therefore in the three components associated with the $3 \mathrm{~B}$ system, the regularization is done by

$$
G_{0}(E) \longrightarrow G_{0}^{(3)}(E) \equiv G_{0}(E)-G_{0}\left(-\mu_{3}^{2}\right),
$$

with the new scale appearing in the regularization of the other 15 components:

$$
G_{0}(E) \longrightarrow G_{0}^{(4)}(E) \equiv G_{0}(E)-G_{0}\left(-\mu_{4}^{2}\right) .
$$

Summarizing our regularizing approach: the physical scales of the four-body problem are the energies of the dimer, one reference trimer, and one reference tetramer. The subtractive procedure adds the regularization parameters $\mu_{3}$ and $\mu_{4}$ to the kernel of the FY equations, which are correlated to the physical three- and four-body scales. The dependence on the subtraction points is eliminated in favor of two observables, which in our case are the values of the trimer and tetramer reference energies. The elimination of the dependence on the subtraction point is possible as evidenced by the existence of correlations between four-boson $s$-wave observables. When presented in terms of dimensionless quantities, the correlation between two consecutive tetramer energies is indeed given by a limit cycle. This correlation, presented in Ref. [26] and further explored in the present work, is in fact consistent with results obtained by other models.

The present four-body regularization strategy keeps fixed the minimal requirement of the three-body properties, while the further freedom is used to introduce the new scale. Therefore, in the regularization procedure of Eqs. (19), by introducing the required three- and four-body regularization parameters as explained above and using Eqs. (22) and (23), we obtain the corresponding zero-range subtracted FY equations, which are given by the following:

$$
\begin{aligned}
\mathcal{K}\left(u_{2}, u_{3}\right)= & 4 \pi \tau\left(\mathcal{E}_{u}\right) \int_{0}^{\infty} d u_{2}^{\prime} u_{2}^{\prime 2} \int_{-1}^{1} d x\left[G_{0}^{(3)}\left(\Pi_{1}\left(u_{2}^{\prime}, u_{2}, x\right), u_{2}^{\prime}, u_{3}\right) \mathcal{K}\left(u_{2}^{\prime}, u_{3}\right)\right. \\
& +\frac{1}{2} \int_{-1}^{1} d x^{\prime} G_{0}^{(4)}\left(\Pi_{1}\left(u_{2}^{\prime}, u_{2}, x\right), \Pi_{2}\left(u_{2}^{\prime}, u_{3}, x^{\prime}\right), \Pi_{3}\left(u_{2}^{\prime}, u_{3}, x^{\prime}\right)\right) \mathcal{K}\left(\Pi_{2}\left(u_{2}^{\prime}, u_{3}, x^{\prime}\right), \Pi_{3}\left(u_{2}^{\prime}, u_{3}, x^{\prime}\right)\right) \\
& \left.+\frac{1}{2} \int_{-1}^{1} d x^{\prime} G_{0}^{(4)}\left(\Pi_{1}\left(u_{2}^{\prime}, u_{2}, x\right), \Pi_{4}\left(u_{2}^{\prime}, u_{3}, x^{\prime}\right), \Pi_{5}\left(u_{2}^{\prime}, u_{3}, x^{\prime}\right)\right) \mathcal{H}\left(\Pi_{4}\left(u_{2}^{\prime}, u_{3}, x^{\prime}\right), \Pi_{5}\left(u_{2}^{\prime}, u_{3}, x^{\prime}\right)\right)\right], \\
\mathcal{H}\left(v_{2}, v_{3}\right)= & 4 \pi \tau\left(\mathcal{E}_{v}\right) \int_{0}^{\infty} d v_{3}^{\prime} v_{3}^{\prime 2}\left[G_{0}^{(4)}\left(v_{3}, v_{2}, v_{3}^{\prime}\right) \mathcal{H}\left(v_{2}, v_{3}^{\prime}\right)\right. \\
& +\int_{-1}^{1} d x G_{0}^{(4)}\left(\left(v_{3}, \Pi_{6}\left(v_{2}, v_{3}^{\prime}, x\right), \Pi_{7}\left(v_{2}, v_{3}^{\prime}, x\right)\right) \mathcal{K}\left(\Pi_{6}\left(v_{2}, v_{3}^{\prime}, x\right), \Pi_{7}\left(v_{2}, v_{3}^{\prime}, x\right)\right)\right] .
\end{aligned}
$$


The physical picture behind the regularization leading to (24) can be described as follows. The three-body scale parameterizes the short-range physics in the virtual propagation of the interacting three-boson subsystem within the four-body system. The four-body scale parameterizes the short-range physics beyond the three-body scale, explored in the four-boson virtual-state propagation between two different fully interacting three-body or disjoint two-body clusters. It is worthwhile to stress that the first term in the right-hand side of the equation for $\mathcal{K}\left(u_{2}, u_{3}\right)$ is the three-boson bound-state equation, when all the other terms are dropped. Thus it is expected that such a term should carry the $3 \mathrm{~B}$ scale, while one could think that the other terms do not require regularization. However, as shown in [15], all the other terms still need to be regularized in order to avoid the 4B ground-state collapse. Although the same regularizing parameter could also be used in all the terms, the freedom to choose it differently is in our hands; the constraint of introducing the trimer properties does not require any further assumption but the given form of the first term in the right-hand side of the equation for $\mathcal{K}\left(u_{2}, u_{3}\right)$. The physical conditions in cold atom traps that allow us to explore a short-range four-boson independent scale, when approaching the Feshbach resonance, are discussed in Ref. [26].

\section{BINDING ENERGIES OF UNIVERSAL TRIMERS AND TETRAMERS}

In this section, before considering our numerical results obtained for the FY bound-state equations (24), we provide a brief discussion on scaling functions, starting with the threeboson case (next section) and moving to tetramer systems, in Sec. III B. After we set up the concept of a scaling function, we present detailed numerical results for the tetramer binding energies, with a comparison with other recently available calculations. The Lanczos-type procedure for solution of the coupled FY equations is shown in Appendix C, where we also give some details on the stability and convergence of our numerical approach.

\section{A. Trimers in the scaling limit}

The three-body system is sensitive to the physics at short ranges, which is parameterized by $\mu_{3}$. After this scale was recognized [2], it was shown that the change of the threebody scale in respect to the two-body one (e.g., 1/a) can be clearly revealed by expressing the energies of the Efimov trimers in a single curve, which defines a scaling function $\mathcal{F}_{3}^{(N)}$, which allows the energies of the full sequence of weakly bound trimers to be built. Thus the energies of successive trimers are correlated by [2]

$$
\sqrt{\frac{B_{3}^{(N+1)}-\bar{B}_{2}}{B_{3}^{(N)}}} \equiv \mathcal{F}_{3}^{(N)}\left( \pm \sqrt{\frac{B_{2}}{B_{3}^{(N)}}}\right),
$$

written in terms of dimensionless quantities. For convenience, in (25) we define $\bar{B}_{2} \equiv B_{2}$ for bound two-body systems (plus sign, or $a>0$ ) and $\bar{B}_{2} \equiv 0$ for virtual states (minus sign, or $a<0)$.
Few cycles are enough to reach a universal function independent on $N$; i.e., in the limit $N \rightarrow \infty$ it reaches a renormalization-group invariant limit cycle [1]. At the critical values, $B_{3}^{(N+1)}=B_{2}$ (for bound two-body) and $B_{3}^{(N+1)}=0$ (for virtual two-body), the scaling function (25) vanishes: $\mathcal{F}_{3}^{(N)}\left( \pm \sqrt{\frac{B_{2}}{B_{3}^{(N)}}}\right)=0$. In this limit, the solution for a bound two-body system $(+$ sign $)$ is given by $B_{3}^{(N)} \simeq 6.925 B_{2}$, and the solution for a virtual two-body system ( - sign) is given by $B_{3}^{(N)} \simeq 1141 B_{2}^{v}$, where $B_{2}^{v}$ is the virtual state energy [2,49]. We observe that the scaling law (25) is one among many possible model-independent correlations between three-body observables for short-ranged interactions. We should also point out that range effects can become relevant as $|a| / r_{0}$ decreases. This is evidenced by the finite-range numerical results from other authors, presented in the scaling plot displayed in Ref. [2]. (On range effects and universal properties of three-body systems, see also Ref. [50].) Therefore range effects will affect Eq. (25) and the above critical values of $B_{3}^{(N)}$.

Evidence of Efimov cycles, with a period of 22.7, in the values of the two-body scattering length $a$ at the peak of the three-body recombination were observed in a cold-atom experiment by the Innsbruck group [22], as well as in other recent experiments reported in [9]. These results gave a response to a quite old discussion on the possible realization of the Efimov effect in real physical systems. The Efimov effect was formulated in the nuclear physics context, when considering the solutions of the three-body Faddeev equations in the limit of infinitely large two-body scattering lengths. Since then, the possible existence of Efimov states in nature have been considered in several works, where the most promising one was the calculations done by Cornelius and Glöckle for a system with three atoms of ${ }^{4} \mathrm{He}$ [51]. Discussions of this matter were revived recently with the advances in cold-atom laboratories, where by using Feshbach resonance techniques it is possible to vary the two-body scattering length from zero to infinite (positive or negative) values.

\section{B. Tetramers in the scaling limit}

Following a complete analogy with the three-body case, we now show evidence for the existence of a limit cycle for the binding energies of successive tetramers. Therefore by considering a shallow dimer (bound or virtual), we introduce a scaling function for the energies of two successive tetramers between two trimers, as

$$
\sqrt{\frac{B_{4}^{(N+1)}-B_{3}}{B_{4}^{(N)}}} \equiv \mathcal{F}_{4}^{(N)}\left(\sqrt{\frac{B_{3}}{B_{4}^{(N)}}} ; \pm \sqrt{\frac{B_{2}}{B_{3}}}\right) .
$$

For the present purpose, the calculations are restricted to bound tetramers below the ground trimer, although the results are valid for tetramers attached to different trimers [26].

The scaling function (26) has the dependence on a fourbody scale independent of the three- and two-body ones. Due to the flexibility of our model it is transparent how to obtain it. The FY equations (24) are solved for $\mu_{3}$ fixed while $\mu_{4}$ is varied. In the unitary limit, the solutions of (24) depend only on the ratio $\mu_{4} / \mu_{3}$, with observables given in units of 
$\mu_{3}$. Eliminating the dependence of the tetramer energies on the ratio $\mu_{4} / \mu_{3}$ by writing the energy of the $(N+1)$ th state as a function of the energy of the $N$ th tetramer, the scaling function (26) is constructed, where the dependence on $\mu_{3}$ is removed in favor of $B_{3}$. As no other scales are present in (24), a scaling function such as (26) seems possible. It remains to be seen whether Eq. (26) is independent of $N$, which is discussed in the next section.

We observe that tetramers below a generic trimer are constrained to be between the two successive Efimov trimers. However, below the ground-state trimer the tetramer collapses as $\mu_{4} / \mu_{3} \rightarrow \infty$ [15], as it is exemplified in Table I(A), for scale ratios above 200 in the unitary limit.

\section{Numerical results close to the unitary limit}

The numerical calculations with the renormalized zerorange model require a definition of the relevant scales. They correspond to the two-, three-, and four-body scales given by $a^{-1}, \mu_{3}$, and $\mu_{4}$, and obviously for a fixed scattering length and trimer scale one can move $\mu_{4}$ to investigate its effect on the spectrum of tetramer ground and excited states.

In Table I we listed our numerical results for tetramer ground- and excited-state binding energies. In part (A) the results at unitary limit $B_{2}=0$ are given for different scale ratios from $\mu_{4} / \mu_{3}=1-400$. According to the obtained results for tetramer binding energies, $\mu_{4} / \mu_{3} \simeq 1.6$ is the threshold for the first tetramer excited state, $\mu_{4} / \mu_{3} \simeq 21$ is the threshold for second tetramer excited state, and close to $\mu_{4} / \mu_{3} \approx 240$ for the third tetramer excited state should appear. At these critical values of scale ratios where $B_{4}^{(N+1)}=B_{3}$ with $B_{2}=0$, the scaling function (26) vanishes,

$$
\mathcal{F}_{4}^{(N)}\left(\sqrt{\frac{B_{3}}{B_{4-c}^{(N)}}} ; 0\right)=0,
$$

with the solutions approaching $B_{4-c}^{(N)} \simeq 4.6 B_{3}$ for each cycle, i.e., $N=0$ and 1 , as we have calculated up to three tetramers below the trimer. The limit cycle is approached quite fast, as verified in the three-boson case [2]. In the critical condition (27) we have one tetramer at the atom-trimer scattering threshold, which allows for a resonant atom-trimer relaxation in trapped cold-atom gases at the Feshbach resonance.

In Tables $\mathrm{I}(\mathrm{B})$ and $\mathrm{I}(\mathrm{C})$, we show the numerical results for positive and negative scattering lengths, respectively, with $\sqrt{B_{2} / B_{3}}= \pm \sqrt{0.02}$. From these results it is clear that by comparing with the case of infinite scattering length and for the same scale ratios, the tetramer binding energies for positive and negative scattering lengths have smaller and larger values, respectively. The critical condition for atom-trimer resonant relaxation near the Feshbach resonance has to be corrected as

$$
\mathcal{F}_{4}^{(N)}\left(\sqrt{\frac{B_{3}}{B_{4-c}^{(N)}}} ; \pm \sqrt{\frac{B_{2}}{B_{3}}}\right)=0,
$$

and $a= \pm 1 / \sqrt{B_{2}}$ does not vanish. By performing a linear expansion around $a^{-1}=0$, from the results given in Tables I(B) and $\mathrm{I}(\mathrm{C})$ one has

$$
B_{4-c}^{(N)} \approx 4.6 B_{3}\left[1-0.8\left(a \sqrt{B_{3}}\right)^{-1}\right] .
$$

TABLE I. Binding energies of ground and excited tetramer states for different four-body scales and for (A) $B_{2}=0$; (B) bound dimer, with $B_{2}=0.02 B_{3}$; and $(\mathrm{C})$ virtual dimer, with $\sqrt{B_{2}}=-\sqrt{0.02 B_{3}}$. In (A) we verify that a third excited tetramer emerges for $\mu_{4} / \mu_{3} \approx$ 240. In (B) and (C), for nonzero two-body binding, we have

\begin{tabular}{|c|c|c|c|c|}
\hline & $\mu_{4} / \mu_{3}$ & $B_{4}^{(0)} / B_{3}$ & $B_{4}^{(1)} / B_{3}-1$ & $B_{4}^{(2)} / B_{3}-1$ \\
\hline \multirow{13}{*}{ (A) } & 1 & 3.10 & & \\
\hline & 1.6 & 4.70 & $7.10 \times 10^{-4}$ & \\
\hline & 5 & 12.5 & 0.531 & \\
\hline & 10 & 24.6 & 1.44 & \\
\hline & 21 & 63.5 & 3.62 & $3.20 \times 10^{-4}$ \\
\hline & 40 & 184 & 7.65 & 0.203 \\
\hline & 50 & 275 & 9.80 & 0.365 \\
\hline & 70 & 520 & 12.9 & 0.629 \\
\hline & 100 & $1.04 \times 10^{3}$ & 20.5 & 1.17 \\
\hline & 200 & $4.06 \times 10^{3}$ & 50.8 & 2.86 \\
\hline & 300 & $9.11 \times 10^{3}$ & 102 & 4.53 \\
\hline & 400 & $1.62 \times 10^{4}$ & 153 & 6.28 \\
\hline & \multicolumn{2}{|c|}{$\mu_{4} / \mu_{3}$} & $B_{4}^{(0)} / B_{3}$ & $B_{4}^{(1)} / B_{3}-1$ \\
\hline \multirow{10}{*}{ (B) } & \multicolumn{2}{|c|}{1} & 2.66 & \\
\hline & \multicolumn{2}{|c|}{1.76} & 4.24 & $9.8 \times 10^{-4}$ \\
\hline & \multicolumn{2}{|c|}{5} & 10.0 & 0.421 \\
\hline & \multicolumn{2}{|c|}{20} & 45.9 & 2.77 \\
\hline & \multicolumn{2}{|c|}{40} & 139 & 6.10 \\
\hline & \multicolumn{2}{|c|}{80} & 506 & 13.0 \\
\hline & \multicolumn{2}{|c|}{200} & $2.86 \times 10^{3}$ & 39.5 \\
\hline & \multicolumn{2}{|c|}{300} & $6.00 \times 10^{3}$ & 69.3 \\
\hline & \multicolumn{2}{|c|}{400} & $9.81 \times 10^{3}$ & 104 \\
\hline & \multicolumn{2}{|c|}{$\mu_{4} / \mu_{3}$} & $B_{4}^{(0)} / B_{3}$ & $B_{4}^{(1)} / B_{3}-1$ \\
\hline & \multicolumn{2}{|c|}{1} & 3.62 & \\
\hline & \multicolumn{2}{|c|}{1.7} & 5.91 & 0.014 \\
\hline & \multicolumn{2}{|c|}{5} & 15.4 & 0.658 \\
\hline & \multicolumn{2}{|c|}{20} & 74.8 & 4.18 \\
\hline (C) & \multicolumn{2}{|c|}{40} & 236 & 9.46 \\
\hline & \multicolumn{2}{|c|}{80} & 873 & 20.6 \\
\hline & \multicolumn{2}{|c|}{200} & $5.02 \times 10^{3}$ & 64.5 \\
\hline & \multicolumn{2}{|c|}{300} & $1.06 \times 10^{4}$ & 115 \\
\hline & \multicolumn{2}{|c|}{400} & $1.73 \times 10^{4}$ & 174 \\
\hline
\end{tabular}
presented results only for the ground and first excited-state binding energies.

The dependence of the tetramer energies with the ratio $\mu_{4} / \mu_{3}$ in the unitary limit is presented in Fig. 2 by using results of Table $\mathrm{I}(\mathrm{A})$. The collapse of the tetramer states by increasing the short-range four-body momentum scale with respect to the three-body one is seen. As for $1 / a=0$, only two scales define the tetramer state; the four-boson binding increases with respect to the trimer one by either increasing $\mu_{4}$ or decreasing $\mu_{3}$. The three tetramer states for $N=0,1$, and 2 show an increase of the binding energies roughly as $\mu_{4}^{2}$. A similar scaling appears for the trimer binding energy with $\mu_{3}^{2}$. The ratio of $B_{4} / B_{3}$ gives the slope of the Tjon line, and it is not a constant as thought previously. As a matter of fact, it can be considered as a parameter measuring the four-body scale. 


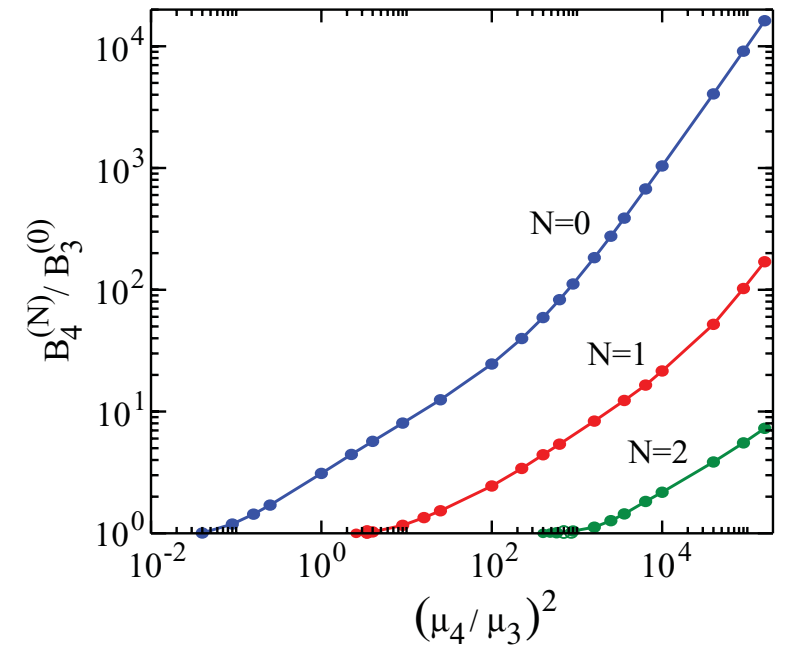

FIG. 2. (Color online) The first three tetramer energy levels at unitary limit, in units of the trimer ground state, are shown as functions of $\left(\mu_{4} / \mu_{3}\right)^{2}$, where $\mu_{4}$ and $\mu_{3}$ are, respectively, the four- and threebody regularizing scaling parameters.

In order to compare directly the numerical results for the three- and four-body limit cycle, we present the Fig. 3 with two panels, (a) and (b). The three-body Efimov states are shown in (b), with the energies given in units of the trimer scaling parameter $\mu_{3}^{2}$. The corresponding four-body Efimov-like states are given in (a), when considering a ground-state trimer in the unitary limit. The dependencies of $B_{4}^{(N)}$ with $B_{3}$ are given with the energies in units of the tetramer energy parameter $\mu_{4}^{2}$ for $N=0,1$, and 2 (with the fixed trimer parameter $\left.\mu_{3}=1\right)$. We illustrate the main qualitative phenomenon of tetramers emerging from the atom plus trimer threshold: by decreasing $B_{3}$, an increasing number of tetramers become bound. A similar phenomenon is shown in the panel (b) for trimers in terms of a dimer energy, which is the Efimov effect. In our calculations we first consider tetramers below a trimer in the ground state, such that the tetramer spectrum is not limited from below and can collapse as we increase the four-body scale in relation to the three-body one. In this case, the number of excited tetramer levels can increase with no limit. However, for a given general excited trimer, the applicability of our results is restricted to a region where the trimer energy varies by a factor of about 515 (the Efimov ratio in the unitary limit). Therefore, between two successive trimer states at most three tetramers can be found.

The results for the four-boson binding energies, plotted in Fig. 1(a), exhibit a limit cycle, which expresses the universal behavior of the energies with a moving four-body scale parameter. The curves shown in this figure reduce to a single curve when they are plotted as the correlation between successive tetramer energies, as presented in Fig. 4 for $M=4$, where we consider the scaling plot in the unitary limit. The scaling function $\mathcal{F}_{4}^{(N)}\left(\sqrt{B_{3} / B_{4}^{(N)}} ; 0\right)$ was built considering up to the third excited state. As in the case of trimer Efimov cycles, the numerical results for tetramers also present a very rapid convergence toward a four-boson limit cycle. For comparison, we also present $\mathcal{F}_{3}^{(N)}\left(\sqrt{B_{2} / B_{3}^{(N)}}\right)$, which has the
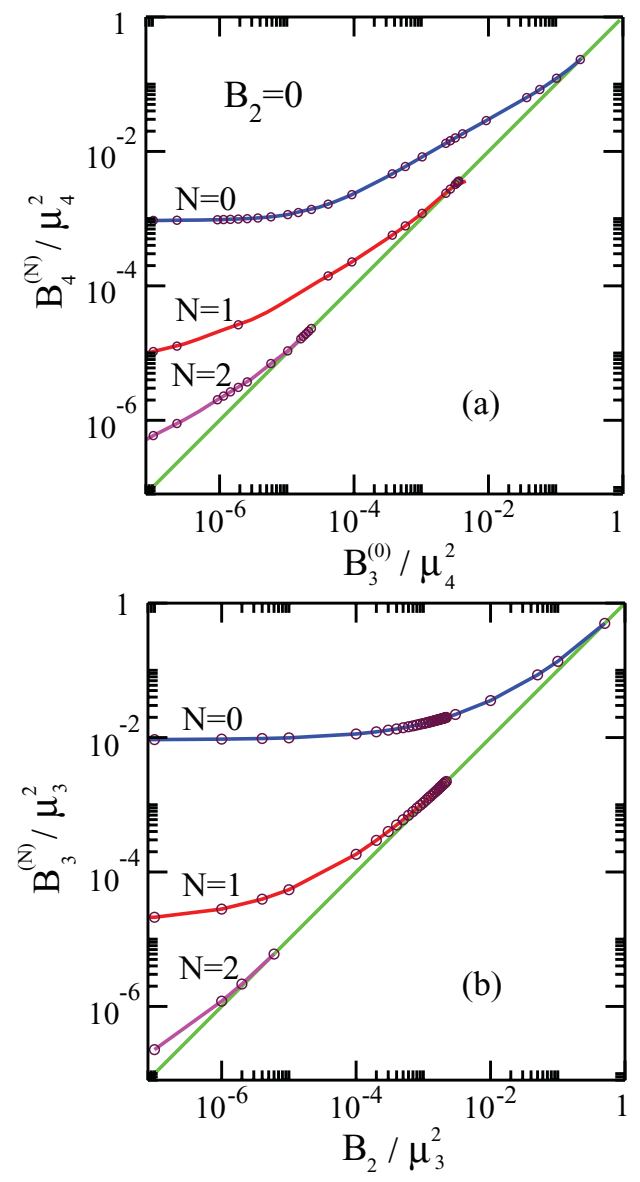

FIG. 3. (Color online) In (a) we have the tetramer energy levels $N=0,1,2$, as functions of the trimer ground-state energy for $B_{2}=0$. For comparison, in (b) we have the first Efimov trimer levels as functions of $B_{2}$. The energy units are $\mu_{4}^{2}$ in (a) (where $\mu_{3}^{2}=1$ ) and $\mu_{3}^{2}$ in (b). In both cases, the threshold is given by the diagonal line.

value of $1 / 22.7$ when $B_{2}$ vanishes. The three-body scaling function, identified inside the plot by $M=3$, was derived in Ref. [2], being compared directly with the corresponding four-body scaling function, identified by $M=4$. The dashed part of the tetramer scaling curve presented in Fig. 4, where $\sqrt{B_{3} / B_{4}^{(N)}}<1 / 22.7$, is not accessible for the excited tetramer energies belonging to the tetramer spectrum that is obtained for a given excited trimer. This restriction implies that a meaningful infinite number of tetramer levels is only possible when considering the trimer in the ground-state level.

Let us consider the unitary limit (when we have the geometrically spaced trimer levels), to resume the global picture suggested by our model. By increasing the tetramer scale with respect to the trimer one, as a new excited tetramer emerges from the threshold, the less excited ones will slide down, moving in the direction of the next deeply bound trimer. Actually these tetramers, between two trimers, are resonances decaying to an atom and a trimer. They will dive into the complex plane through the atom-trimer scattering cut with their width increasing as the four-body scale is incremented. This qualitative discussion deserves further investigation from the point of view of the complex analytical structure of the four-body scattering equations. Our picture suggests that, 


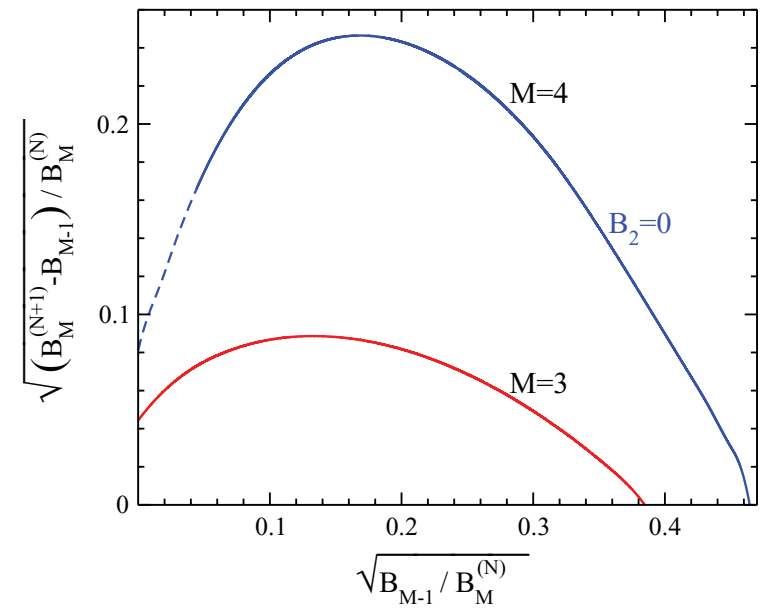

FIG. 4. (Color online) The four- and three-body scaling functions are represented, respectively, by $M=4$ and $M=3$. The energies are rescaled in terms of $B_{M}^{N}$, where $N$ represents the corresponding energy level in both cases $(N=0$ is the ground state). The behavior of the excited trimer $(M=3)$ and tetramer $(M=4)$ energies is shown as the corresponding subsystem energy $\left(B_{2}\right.$ or $\left.B_{3}\right)$ varies. In the case of $M=4, B_{2}$ is fixed to zero and the dashed part is only reachable by tetramers below the ground-state trimer.

within the unitary limit, at most three tetramer resonances lie between two successive trimers.

In Fig. 5, to verify the universality of the four-boson scaling function (26) and for comparison, we include results obtained

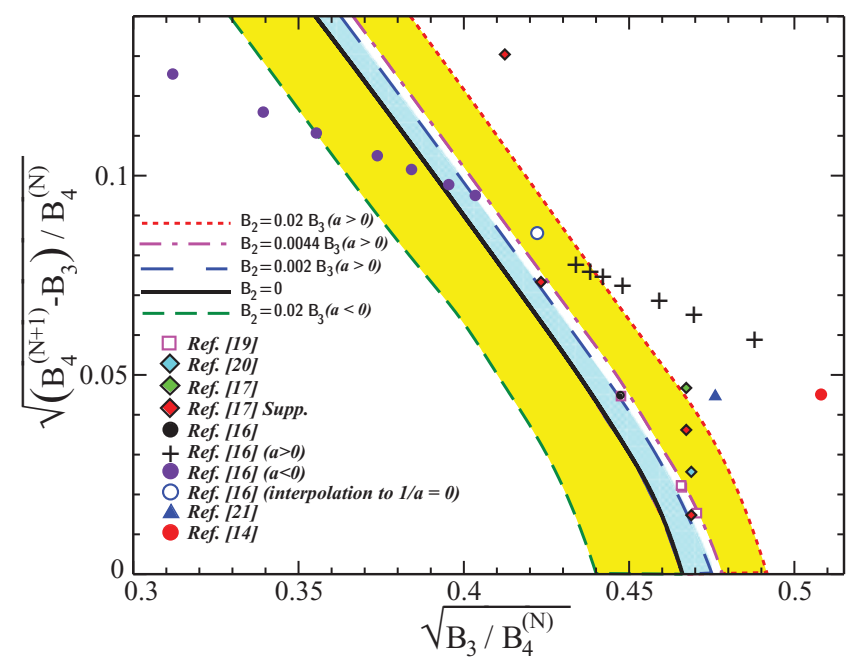

FIG. 5. (Color online) Our results for the four-boson scaling function, reported in [26], are displayed in the limited region where more recent results are available. The plots are shown for $B_{2}=0$ (solid-black curve) and in four cases with $B_{2} \neq 0$ [three cases with bound dimers, $B_{2} / B_{3}=0.02,0.0044,0.002$, and one case with a virtual dimer, $\sqrt{B_{2} / B_{3}}=-\sqrt{0.02}$ ], as indicated inside the frame. In the case of $B_{2}=0$, this figure refers to the right-hand-side corner of Fig. 4 for $M=4$. The results were explicitly verified numerically for $N=0$ and $N=1$, considering the energies $B_{4}^{N=0,1,2}$ in case $B_{2}=0$; and for $N=0$ in the other cases where $B_{2} \neq 0$. The symbols refer to other model calculations (as indicated) near the unitary limit.
TABLE II. Binding-energy ratio of tetramer ground and excited energies to a trimer level $\left[B_{4}^{(0)} / B_{3}, B_{4}^{(1)} / B_{3}-1\right]$, where the results of Refs. $[17,19]$ refer to different trimers.

\begin{tabular}{lcccc}
\hline \hline Ref. [52] & Ref. [14] & Ref. [21] & Ref. [16] & Ref. [20] \\
\hline$[4.46,0.06]$ & {$[4.075,0.003]$} & {$[4.41,0.01]$} & {$[5.0,0.01]$} & {$[4.55,0.003]$} \\
Ref. [17] & & Ref. [19] \\
\hline$[4.58,0.01]$ & & {$[4.5175,0.00106]$} \\
{$[5.88,0.10]$} & {$[4.6041,0.00217]$} \\
{$[4.58,0.006]$} & {$[4.6104,0.00227]$} \\
{$[5.58,0.03]$} & & {$[4.6108,0.00228]$} \\
{$[4.55,0.001]$} & & {$[4.9929,0.00997]$} \\
- & & {$[4.6114,0.00228]$} \\
\hline
\end{tabular}

in other recent calculations. ${ }^{1}$ In Table II we have listed the available results of other authors for binding-energy ratios of ground and excited tetramers close to the unitary limit. Within those results we should also point out that the ones given in Refs. [17,19] are for more than one trimer level. In our results with zero-range interaction, we include not only the exact limit $B_{2}=0$, but also nonzero two-body energies (bound or virtual), as shown in Tables $\mathrm{I}(\mathrm{A})-\mathrm{I}(\mathrm{C})$. The sensibility of the scaling function with variation of the two-body conditions is shown when nonzero two-body energies are used. As it is shown, the exact unitary limit scaling plot is shifted to the right-hand side (left-hand side) when considering nonzero two-body bound (virtual) state energies.

The results of available calculations for tetramer energies plotted in the way we are suggesting put in evidence the effect of the four-body scale. Consistent with our findings, they slide along the universal correlation shown in Fig. 5. The sensitivity of our defined scaling plot, with respect to variations of a twobody observable such as the scattering length or range effects, is also consistent with the results obtained by other authors.

In order to verify the sensitivity of the results (obtained for $B_{2}=0$ ) with small changes in the dimer energies, we calculate the corresponding scaling functions for a few cases with $B_{2} \neq 0$. In the given results, we have three cases for bound two-body systems, where $B_{2} / B_{3}=0.02,0.0044$, and 0.002 , and one case for a virtual two-body state with $\sqrt{B_{2} / B_{3}}=$ $-\sqrt{0.02}$. In Fig. 5 we have also included the Hammer and Platter results for both a virtual and real dimer close to unitary limit (see Fig. 2 of Ref. [16]). Despite the observed difference in the position of both results in our plot, it is remarkable that they both fit nicely in our scaling curve, meaning that the four-boson model of Ref. [16] is sensitive to a four-body scaling parameter, carried out implicitly by the momentum cutoffs used in their calculations.

Although we calculate tetramer energies below the trimer ground state, such that the dashed part of Fig. 4 is also verified, the results for tetramers attached to any other Efimov state do not change the present conclusions on the existence of a

${ }^{1}$ For an update of Fig. 5, with more recent results of other authors, see T. Frederico, A. Delfino, M. R. Hadizadeh, L. Tomio, and M. T. Yamashita, e-print arXiv:1201.6586. 
proper four-body scale or its universal manifestation through the scaling function (26), calculated with the zero-range model regularized within our scheme. This fact is evidenced in Fig. 5. Distinct, short-ranged interaction models [16,17,19-21] show that the energies of successive tetramers linked to the tower of Efimov states scale according to the plot, thus verifying the universality of the four-boson limit cycle.

\section{STRUCTURE OF UNIVERSAL TETRAMERS}

The effect of the four-body scale on the structure of tetramers at the unitary limit is detailed in this section. We show results for the FY components in momentum space for different scale ratios as well as for momentum probability densities. A close inspection of the reduced FY components, $\mathcal{K}$ and $\mathcal{H}$, will show how $\mu_{4}$ manifests through their dependence on the different Jacobi momenta. An analogous study will be presented for the momentum probability densities.

\section{A. Reduced Faddeev-Yakubovsky components}

The reduced FY components $\mathcal{K}\left(u_{2}, u_{3}\right)$ and $\mathcal{H}\left(v_{2}, v_{3}\right)$ are shown to spread out up to momentum of the order 1 , due to the relevance of the four-body momentum scale $\mu_{4}$ to regularize the kernel of the set of FY equations (24) at short distances. Both components have narrow peaks appearing at small momenta, which are even more pronounced for higher scale ratios $\mu_{4} / \mu_{3}$ and more excited states. They follow a typical tetramer momentum scale, $\sqrt{B_{4}^{(N)}} / \mu_{4}$. These features are evident in Figs. 6 and 7 . For a given ratio $\mu_{4} / \mu_{3}$, the ground-state wave function is more expanded in momentum space in comparison to excited states. The $K$ and $H$ channels, and consequently, the total wave function for smaller tetramer bindings, should extend to larger distances as compared to the corresponding ones with larger binding.

The peaks at small momentum are due to the nearest trimer pole in the three-boson interacting resolvent appearing in the $K$-channel equation (24). This leads to a dominance of $\mathcal{K}$ over $\mathcal{H}$ for small momentum when the tetramer energy approaches $B_{3}$.

In Figs. 6 and 7 we detail the sections of $\mathcal{K}\left(u_{2}, u_{3}\right)$ and $\mathcal{H}\left(v_{2}, v_{3}\right)$ with one of the Jacobi momenta being zero for scale ratios of 5, 50, and 200. The interpretation of the Jacobi momentum with respect to the relevant scales is necessary to proceed in the analysis of these plots. The variable $u_{2}$ is the relative momentum of particle 3 with respect to the center of mass of the pair $(1,2)$, which belongs to a trimer configuration (see Fig. 1). Note that in our derivation of the FY equation for the zero range potential, we have chosen to factor the dependence of the relative momentum of the $(1,2)$ pair in the definition of the reduced amplitudes, as given by Eq. (18). Naively, for kinematical reasons, it is reasonable to expect that, within the tetramer, the average distance between two bosons is larger than the average distance between the center-of-mass (c.m.) of the virtual pair to the third particle, which is larger than the average distance of the virtual trimer to the fourth particle. The ordering effect comes because the clusters, being heavier, should have their c.m. closer to the c.m. of the tetramer. This size ordering has a correspondence with respect to the momentum variables. In $\mathcal{K}\left(u_{2}, u_{3}\right)$, the
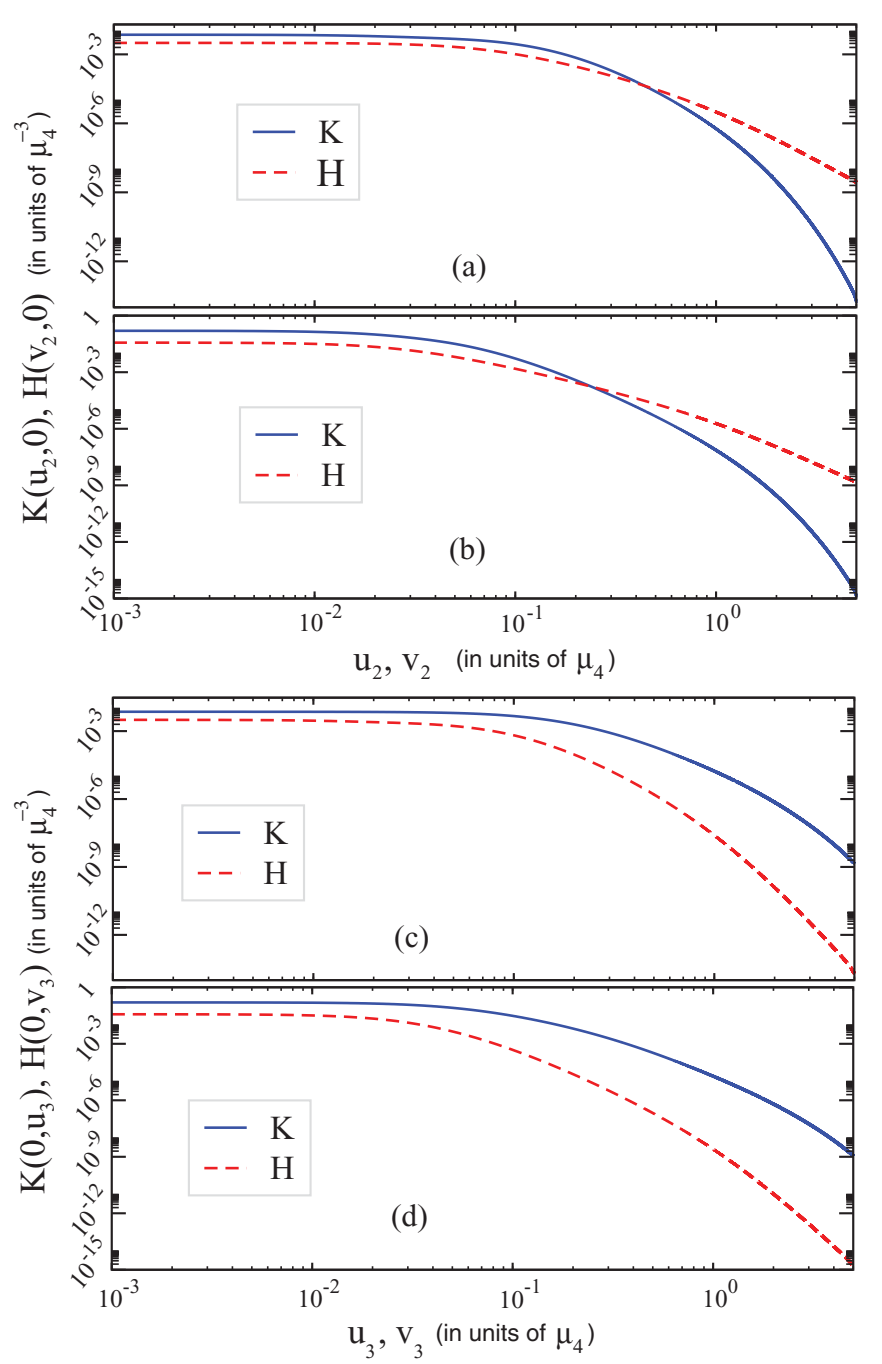

FIG. 6. (Color online) The Yakubovsky components $\mathcal{K}$ and $\mathcal{H}$ as functions of the Jacobi momenta for scale ratio $\mu_{4} / \mu_{3}=5$ when only one four-body excited state exists. In frames (a) and (b) the components are shown as a function of $u_{2}, v_{2}$ where $u_{3}, v_{3}=0$, and in frames (c) and (d) they are shown as a function of $u_{3}, v_{3}$ where $u_{2}, v_{2}=0$. In (a) and (c) we have results for the groundstate level and in (b) and (d) for the first excited-state level. The normalization is such that $\int_{0}^{\infty} d u_{2} u_{2}^{2} \int_{0}^{\infty} d u_{3} u_{3}^{2} \mathcal{K}^{2}\left(u_{2}, u_{3}\right)+$ $\int_{0}^{\infty} d v_{2} v_{2}^{2} \int_{0}^{\infty} d v_{3} v_{3}^{2} \mathcal{H}^{2}\left(v_{2}, v_{3}\right)=1$.

momentum of the fourth particle in respect to the virtual trimer $u_{3}$ should explore larger momentum regions than $u_{2}$, which is the relative momentum of the third particle with respect to a pair. An analogous reasoning suggests that the momentum dependence of $\mathcal{H}\left(v_{2}, v_{3}\right)$ is such that $v_{2}$ explores larger momentum regions than $v_{3}$ (the relative momentum of two bosons). These qualitative properties are verified in Figs. 6 and 7.

The plots in Fig. 6 show the reduced FY components for the ground state, frames (a) and (c), and excited state, frames (b) and $(\mathrm{d})$, for $\mu_{4} / \mu_{3}=5$. The high-momentum tails of $\mathcal{H}\left(v_{2}, 0\right)$ and $\mathcal{K}\left(0, u_{3}\right)$ with respect to $\mathcal{H}\left(0, v_{3}\right)$ and $\mathcal{K}\left(u_{2}, 0\right)$ are visible by comparing frames (a) and (c) in the case of the ground state, and (b) and (d) for the excited state. By inspecting the pair of frames (a) against (b), and (c) against (d), we also 

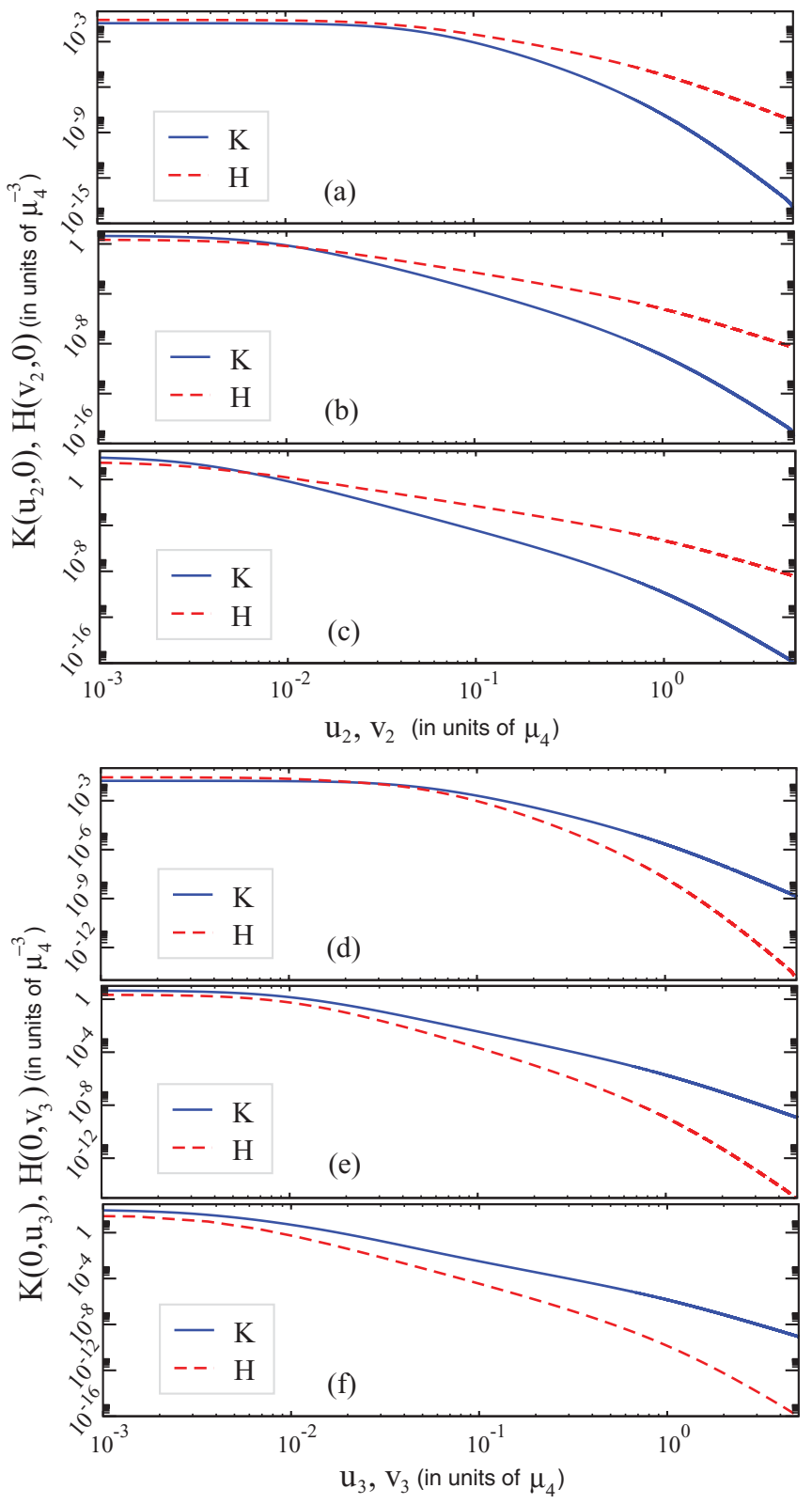

FIG. 7. (Color online) Following the caption of Fig. 6, here we have the same quantities for $\mu_{4} / \mu_{3}=50$. In frames (a) and (d) we have the ground state; in (b) and (e) the first excited state; and in (c) and (f) the second excited state.

note that all the FY components for the excited state are more concentrated at lower momentum than the corresponding ones for the ground state. Although the binding-energy ratio $B_{4}^{(1)} / B_{3}$ for the excited state is close to 1 , the high-momentum tail bringing the four-body scale is present, giving to this state the possibility to move as $\mu_{4}$ is changed with respect to $\mu_{3}$. These qualitative features are supported by the results we have obtained for the larger scale ratio, such as $\mu_{4} / \mu_{3}=50$ and 200 , where three tetramer states are possible. This behavior is clearly shown in Fig. 7 for $\mu_{4} / \mu_{3}=50$.

In all the cases we analyzed, the dependence of $u_{3}$ in $\mathcal{K}\left(u_{2}, u_{3}\right)$ and $v_{2}$ in $\mathcal{H}\left(v_{2}, v_{3}\right)$ show that these two variables explore larger momentum regions than $u_{2}$ and $v_{3}$, respectively. As we have shown, $\mathcal{K}\left(u_{2}, 0\right)$ and $\mathcal{H}\left(0, v_{3}\right)$ have a less prominent tail at large momentum than $\mathcal{K}\left(0, u_{3}\right)$ and $\mathcal{H}\left(v_{2}, 0\right)$. If, for any reason, the high momentum dependence that appears in the $K$ and $H$ channel is lessened, the dependence on the four-body scale will be minimized (may be even completely removed!). Therefore, an unreasonable selection of cut-off values in the mapping of momentum variables, which can happen in particular when a fixed cutoff is being used for increasing values of the scale ratios, can lead to convergence in the four-body binding energies rather than collapse. For the discretization of momentum variables one should not only consider large enough cut-off values, consistent with those used in the four-body scale, but also consider a reasonable number of mesh points in the interval near zero momentum. Since the iteration of the coupled Eqs. (24) requires a very large number of multidimensional interpolations on the Yakubovsky components, we have used cubic-Hermite splines to reach high computational accuracy.

From the above analysis, represented in Figs. 6 and 7, we conclude that, as opposed to the general belief stating that in four-body atomic and nuclear calculations, with model potentials in the FY scheme, the $K$ channel is always dominant, we show some cases where the $H$ channel is dominant even at low momentum. This is shown, in particular, for high scale ratios $\mu_{4} / \mu_{3}$.

\section{B. Momentum probability densities}

The tetramer wave function $\Psi\left(u_{1}, u_{2}, u_{3}\right)$ fully symmetrized is built from Eq. (7) using the definitions (18) of the $K$ and $H$ components in terms of $\mathcal{K}\left(u_{2}, u_{3}\right)$ and $\mathcal{H}\left(v_{2}, v_{3}\right)$. In order to simplify our analysis of the wave function and to obtain insight as to how the momentum is shared among the Jacobi coordinates, we introduce the momentum probability densities $n\left(u_{i}\right)$ as

$$
\begin{aligned}
n\left(u_{i}\right) & =u_{i}^{2} \int_{0}^{\infty} d u_{j} u_{j}^{2} \int_{0}^{\infty} d u_{k} u_{k}^{2} \Psi^{2}\left(u_{1}, u_{2}, u_{3}\right) \\
\int_{0}^{\infty} d u_{i} n\left(u_{i}\right) & =1
\end{aligned}
$$

with $(i, j, k)=(1,2,3),(2,3,1)$, and $(3,1,2)$. For reference we use the variables $u_{i}$ of the $K$ configuration, where $u_{1}$ is the relative momentum of a pair, $u_{2}$ the relative momentum of the third boson to the center of mass (c.m.) of the pair, and $u_{3}$ is the relative momentum of the fourth boson to the c.m. of the other three (see Fig. 1). As we have discussed in Sec. IV A, the average values of the momentum variables are ordered as $\left\langle u_{1}\right\rangle<\left\langle u_{2}\right\rangle<\left\langle u_{3}\right\rangle$, which is reflected in momentum densities, independent of the degree of excitation of the tetramer.

The momentum probability densities $n\left(u_{1}\right), n\left(u_{2}\right)$, and $n\left(u_{3}\right)$ for scale ratio $\mu_{4} / \mu_{3}=50$, for ground, first, and second excited tetramer states, are presented in Fig. 8. In the left panel we show our results for the probability densities in units of $\mu_{4}$. Clearly, for all three momentum densities, the second excited tetramer leads to a very sharp peak close to zero momentum, whereas for the first excited and ground tetramers the peak is more wide, which is evident as the size is inversely correlated to the tetramer binding energy. For the ground tetramer state the FY components and consequently the 4B total wave function are expanded to a higher momentum region, and for excited tetramers they are more restricted to the 

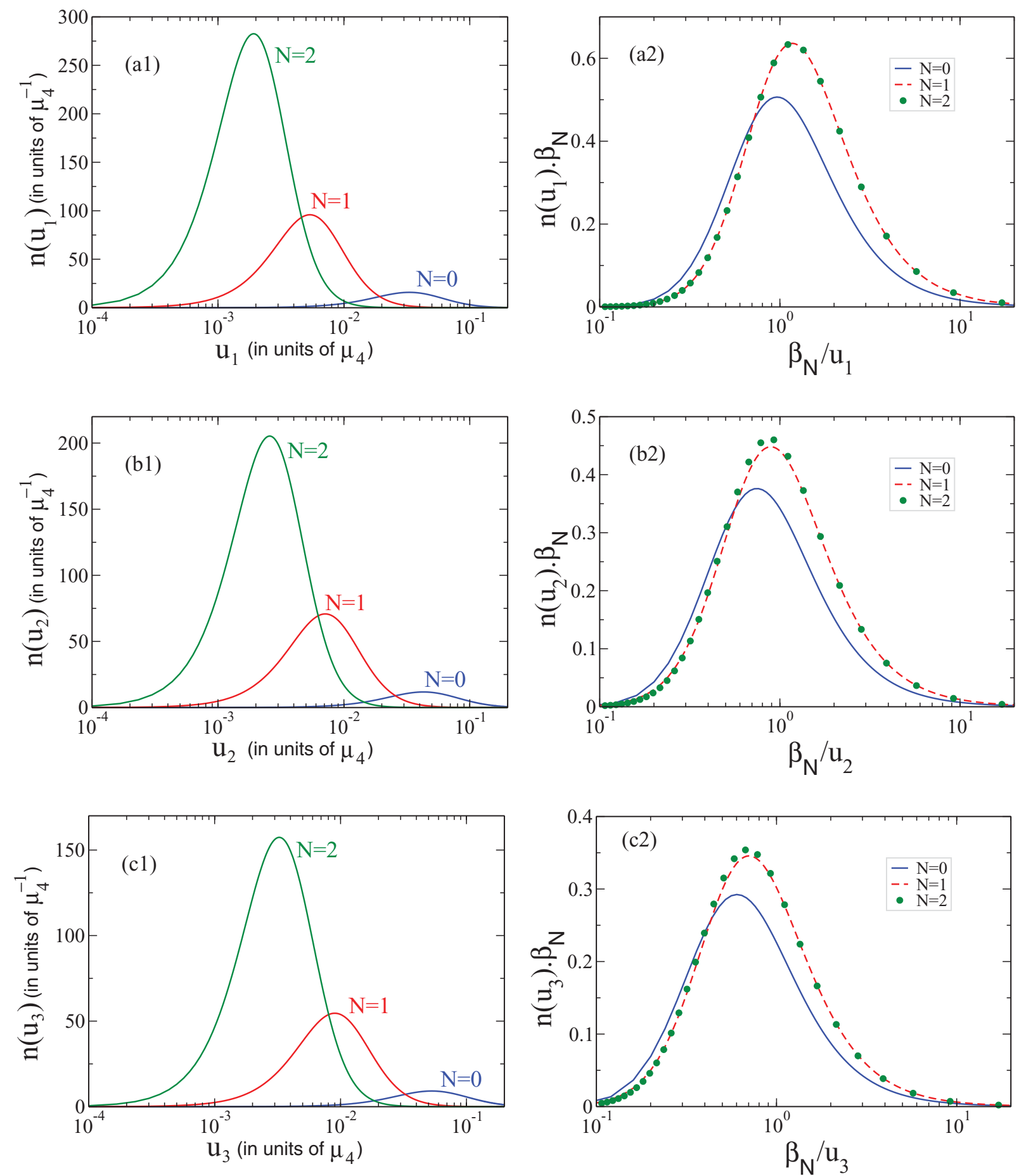

FIG. 8. (Color online) Momentum distribution functions $n\left(u_{1}\right), n\left(u_{2}\right)$, and $n\left(u_{3}\right)$, with scale ratio $\mu_{4} / \mu_{3}=50$, for the ground $(N=0)$ and the first two $(N=1,2)$ excited tetramer levels, normalized as shown in Eq. (30). In the left panels they are given as functions of the corresponding momentum, where all momenta are in units of the momentum scale $\mu_{4}$. In the right panels, the momentum distributions are rescaled by the momentum factor $\beta_{N} \equiv \sqrt{B_{4}^{(N)}}$, where $N$ is the tetramer level.

zero momentum region. In the right panel of Fig. 8 we present the probability densities in momentum units $\beta_{N} \equiv \sqrt{B_{4}^{(N)}}$, for $\mu_{4} / \mu_{3}=50$. By considering this scaling factor corresponding to the energy of each tetramer state, which from Table I varies two orders of magnitude, the densities are amazingly close.
The tetramer energy can be considered as the physical scale, which correlates other observables.

One feature of the universal properties of the four-body wave function is presented in Fig. 9, where we have compared the momentum distribution functions $n\left(u_{i}\right)$ of the two shallowest tetramers for two very different values of $\mu_{4} / \mu_{3}$ equal 


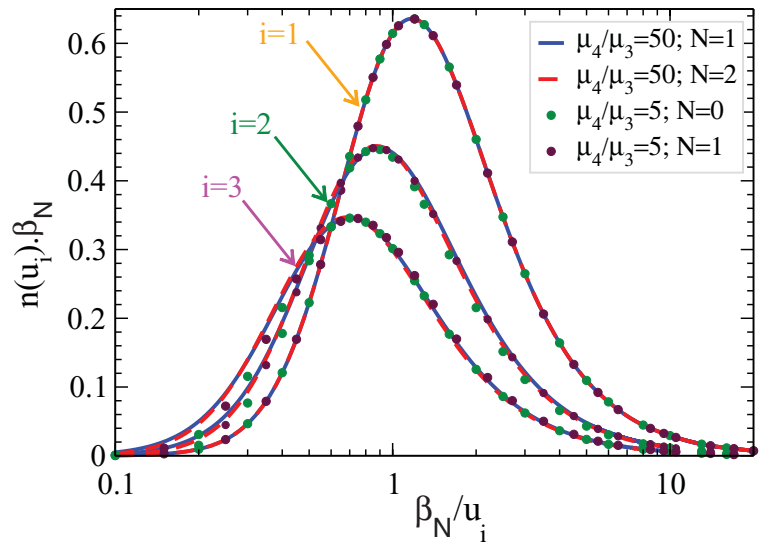

FIG. 9. (Color online) Renormalized momentum distributions as functions of the Jacobi momenta $u_{i=1,2,3}$, considering the scaling ratios $\mu_{4} / \mu_{3}=5$, when there is only one excited state, and $\mu_{4} / \mu_{3}=$ 50 , when we have two excited states. The units are given by the momentum factor $\beta_{N} \equiv \sqrt{B_{4}^{(N)}}$. For each momentum component $u_{i}$, the corresponding distributions of both scale ratios, when renormalized to the same maximum value, are shown to reduce almost to the same final form (the labels inside the frame are just to indicate the performed calculations).

to 5 and 50 . The functions $n\left(u_{i}\right)$ were rescaled to the same maximum value to make transparent their universal form. The comparison between them clearly confirms a universal dependence of the momentum distributions on the tetramer binding energy. Note that these quantities are plotted in terms of dimensionless quantities in natural units of the length associated with the tetramer energy.

In Fig. 9 we have shown the renormalized form of the rescaled distribution functions, considering two different scale ratios $\frac{\mu_{4}}{\mu_{3}}=5$ and 50 , whereas the distributions are renormalized to the same maximum value. As we have shown, the distribution functions have universal shape, independent of the value of scale ratio. We should also add a remark that in order to simplify the presentation of Fig. 9, we have included only the momentum distribution functions for the two more excited states in the case of $\frac{\mu_{4}}{\mu_{3}}=50$, whereas the same universal shape is also obtained for the momentum distribution functions of the ground state (see Fig. 8). The ground-state energy ratio is $B_{4}^{(0)} / B_{3}=275$ (see Table I), which means that $B_{4}^{(0)}$ is about $2.7 \mu_{3}^{2}$, while for the two excited states, $B_{4}^{(1)}$ and $B_{4}^{(2)}$ is much smaller than $\mu_{3}^{2}$. Thus the three-body regularization scale is far more important to the ground state than for the excited states, which approach a universal form for the momentum distribution functions.

\section{Tetramer wave function}

The calculation of the FY amplitudes $K$ and $H$, given by Eq. (18) for form factor unity, with the reduced amplitudes $\mathcal{K}$ and $\mathcal{H}$ from the numerical solution of the zero-range FY integral equations (24), allows the four-boson wave function to be built fully symmetric by boson exchange. The necessary permutations to reconstruct the 4B wave function from the computed $K\left(u_{1}, u_{2}, u_{3}\right)$ and $H\left(v_{1}, v_{2}, v_{3}\right)$ are given by introducing permutation operators $\mathcal{O}$ with the corresponding
FY amplitudes denoted by $K_{\mathcal{O}}$ and $H_{\mathcal{O}}$ (see Appendix B). By computing $K_{\mathcal{O}}$ and $H_{\mathcal{O}}$ for different $\mathcal{O}$, the $4 \mathrm{~B}$ total wave function can be obtained by using Eqs. (B1) or (B2).

For our purposes of presenting graphically the total wave function, we show plots for the angle-averaged wave function, where the dependence on the relative angles between Jacobi momentum $\vec{u}_{i}[i=(1,2,3)]$ is integrated. Our notation is such that we still use $\Psi\left(u_{1}, u_{2}, u_{3}\right)$ for the angle-averaged wave function. Instead of the usual normalization, we consider

$$
\begin{aligned}
\langle\Psi \mid \Psi\rangle & \equiv \int_{0}^{\infty} d u_{1} u_{1}^{2} \int_{0}^{\infty} d u_{2} u_{2}^{2} \int_{0}^{\infty} d u_{3} u_{3}^{2} \Psi^{2}\left(u_{1}, u_{2}, u_{3}\right) \\
& =1 .
\end{aligned}
$$

In Figs. 10-12 we present our numerical results for the angle-averaged total wave function with scale ratio $\mu_{4} / \mu_{3}=$ 50 for the ground, first, and second excited states. The wave functions are shown as functions of two Jacobi momenta, when the third one is chosen to be zero. As we did before, the magnitudes of Jacobi momenta are rescaled by the momentum factor $\beta_{N} \equiv \sqrt{B_{4}^{(N)}}(N=0,1,2)$. In view of the normalization condition (31), this momentum scaling implies a rescaling of the magnitude of the corresponding total wave function by a factor of $\beta_{N}^{9 / 2}$.

The universal form of the $4 \mathrm{~B}$ wave function is evidenced in Figs. $10-12$ by a proper rescaling with the four-body energy. Correspondingly, the contour plots are also presented as functions of two nonvanishing Jacobi momenta, given in units of $\beta_{N}$. Also the momentum density distributions, for two excited states, present a universal form, i.e., independent of the binding energies, when the momentum is measured in units of $\beta_{N}$, as shown in Fig. 8. Note that each of the choices of the vanishing momentum implies a very long wavelength limit for the fourth particle, indicating the dominance of a three-body cluster configuration within the four-body system. This configuration carries the four-body scale information, as clearly shown by the plots on the wave-function dependence on the two nonvanishing momentums in units of $\beta_{N}$.

\section{CONCLUSIONS AND PERSPECTIVES}

The momentum-space structure of the FY components of weakly bound tetramers was thoroughly analyzed at the unitary limit using a renormalized zero-range two-body interaction, with three- and four-body scales. Our regularization scheme allows independent introduction of these two scales, providing a general framework to investigate the universal properties of tetramers, which have extended tails in the classically forbidden region. The step to renormalized results for the observables is found by introducing scaling functions, which are written only in terms of dimensionless physical quantities. These universal scaling functions numerically achieve a limit cycle.

The universal scaling function correlating two successive tetramer energies attached to one trimer comes from the sensitivity of the four-boson system to a short-range fourbody scale. Each excited $N$ th tetramer energy $B_{4}^{(N)}$ moves as the short-range four-body scale changes while the trimer properties are kept fixed. We suggest the ratio of $B_{4} / B_{3}$, which gives the slope of the Tjon line and is not a constant as 

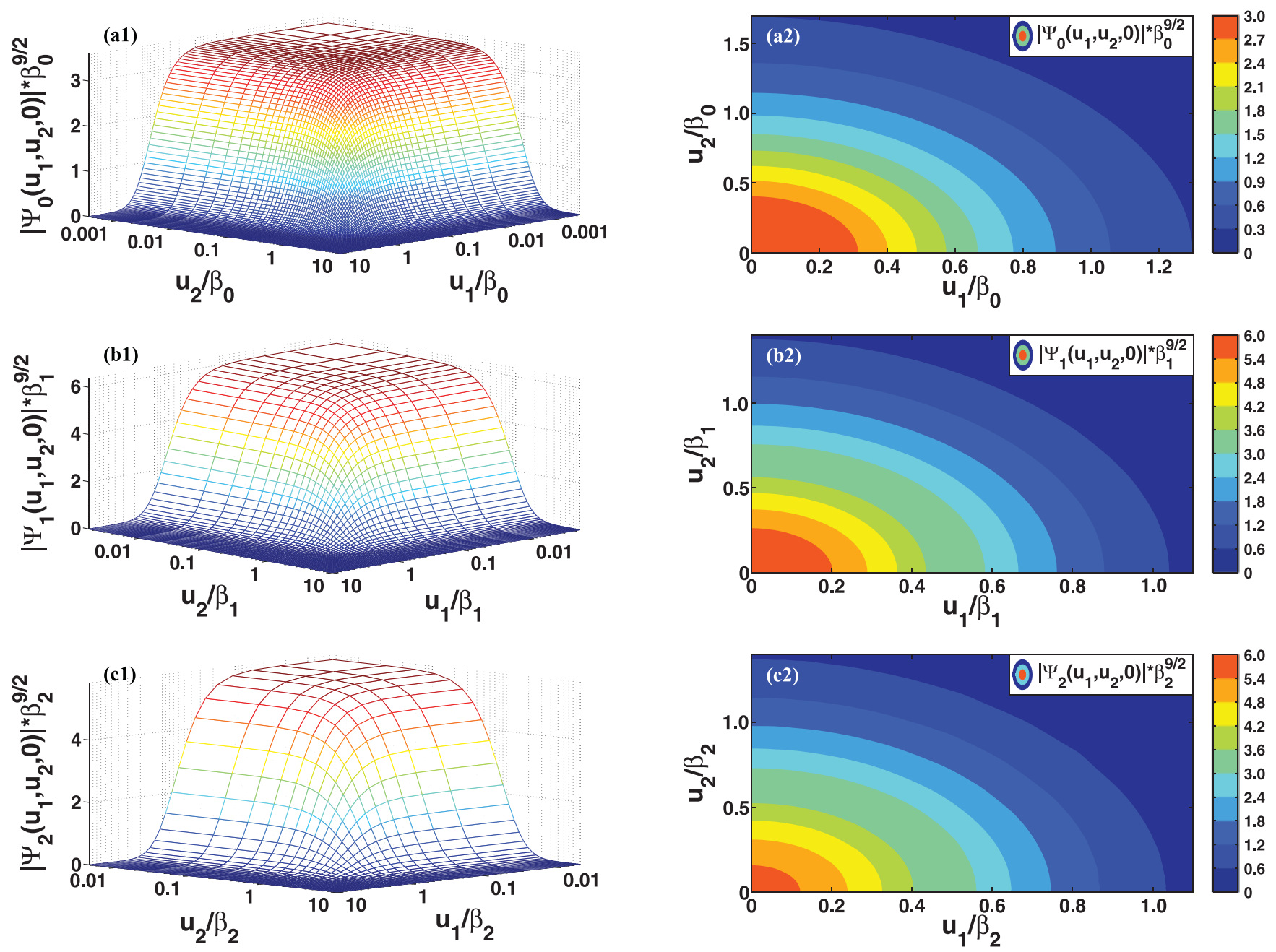

FIG. 10. (Color online) The magnitude of the 4B total wave function $\Psi\left(u_{1}, u_{2}, u_{3}\right)$ as a function of $u_{1}$ and $u_{2}$, with $u_{3}=0$, for the scale ratio $\mu_{4} / \mu_{3}=50$. The corresponding contour plots are shown for the low-momentum region near the peak of the wave function.

thought before, as a parameter measuring the four-body scale. At the unitary limit, the successive $(N+1)$ th tetramer state emerges from the atom plus trimer threshold for a universal ratio $B_{4}^{(N)} / B_{3}=B_{4}^{(N)} / B_{4}^{(N+1)} \simeq 4.6$, which does not depend on $N$. The atom-trimer relaxation resonates when the tetramer hits the scattering threshold.

We also find that other model results obtained at the unitary limit, or close to it $[16,17,19-21]$, are quite consistent with our four-boson scaling plot, giving confidence regarding the universality of our proposed scaling function. As evidenced by our results, the independent behavior of the four-body scale is verified, in particular, when a universal excited four-boson state pumps out from the atom-trimer threshold as the fourboson parameter is driven to short distances or to the ultraviolet momentum region. In the case that both scales are similar, we confirm that our scaling approach is consistent with the results of other model calculations. We note that the results obtained by other groups appear near the threshold region due to the model assumptions that have been considered, which are too restrictive and need to be relaxed to allow a wider variation between the four- and three-body properties. The suggested scaling plot offers a model-independent way to view the relation between successive tetramer states, which can be verified by experiments exploring two-body scattering lengths very close to the Feshbach resonance, where multiboson forces are expected to be active [27]. A four-boson short-range interaction can drive the four-body scale independently from the three-body one. Short-range three-body forces accompanied by different off-shell behaviors of the two-body interaction, which keep the low-energy two- and three-body properties unaltered, could provide another possibility to move the four-body scale in practice.

The four-body scaling plot shows the independent behavior of a four-body scale (for a fixed three-body one) in a similar way that the three-body scaling plot, given in Ref. [2], resumes all the Efimov states obtained by changing the three-body scale. Next, we should emphasize that the scaling curve relating two successive tetramer energies is valid not only for the stable tetramers attached to the ground-state trimer, but also for the unstable tetramers between two successive trimers. For such a conclusion we have also performed resonance calculations of tetramers attached to excited trimers, which are under detailed numerical analysis to be included in a future work. We avoid presenting such results in the present work, mainly because we have considered other independent and precise calculations supporting our claim of 

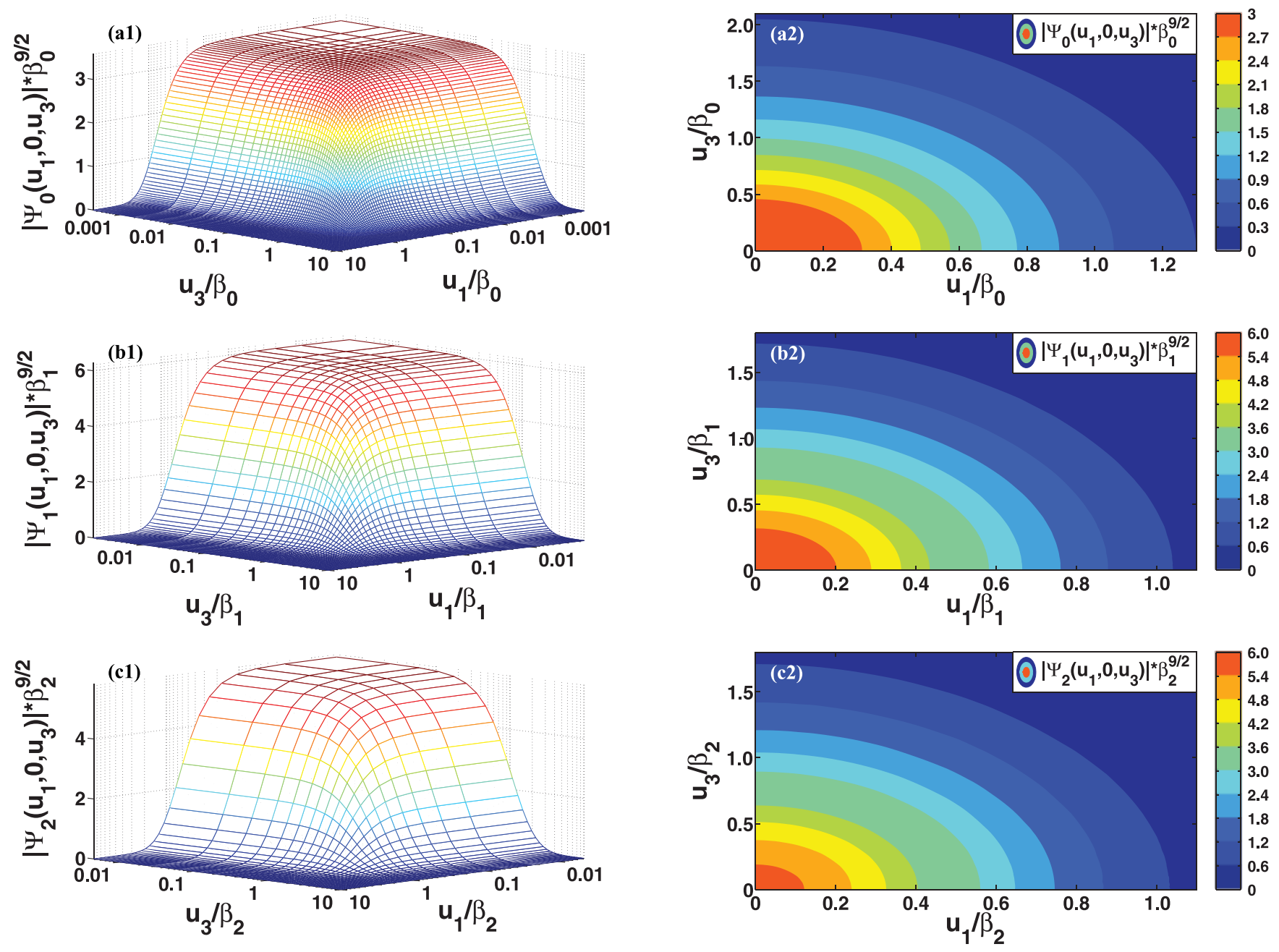

FIG. 11. (Color online) As in Fig. 10, the wave function is given in terms of $u_{1}$ and $u_{3}$, with $u_{2}=0$.

model independence. In this regard, the recent calculations performed by Deltuva [19] are consistent with our results and give confidence on the model independence of the scaling plot relating the energies of two successive tetramers between two successive Efimov trimers in the unitary limit. As we have shown, the effect of scattering length variations is noticeable and shows a universal pattern, in agreement with calculations shown in [16], performed within the context of effective field theory. However, range corrections, which were not yet explored within a general study considering the trimer and tetramer interwoven spectra, will certainly have their importance in explaining how results near the unitary limit deviate from the calculated scaling function for a zero-range force. In this respect, range corrections, linear in the twobody effective range, have already been addressed within an effective theory treatment of the three-boson problem with subtracted momentum-space integral equations [53].

The scaling plot representation exhibiting the dependence on the four-body scale, which is also being confirmed by other models, shows that the model independence is not fortuitous. The reason for that comes from the fact that the wave function of tetramers is largely dominated by configurations where the bosons are outside the potential range in the classically forbidden region and depend just on two scales at the unitary limit. The sensitivity to the short-range scales appears in both the $K$ and $H$ channel of the FY decomposition, which present high-momentum tails for any degree of tetramer excitation. We also found that the $H$ channel is favored over the $K$ channel at low momentum when the four-body momentum scale largely overcomes the three-body scale.

The universal form of the wave function is put forward at the unitary limit by a scaling plot where the probability momentum densities for different Jacobi momenta are shown in units such that the tetramer binding energy is 1 . We found that the shapes are independent of the scale ratio and excitation, depending only on the chosen Jacobi momenta. A simple scaling rule gives the ordering $\left\langle u_{1}\right\rangle<\left\langle u_{2}\right\rangle<\left\langle u_{3}\right\rangle$, where $u_{1}$ is the relative momentum of a pair, $u_{2}$ is the relative momentum of the third particle to the pair, and $u_{3}$ is the relative momentum of the fourth particle in respect to the three-body subsystem.

Our calculations of tetramer binding energies, with corresponding structure of momentum probability density and wave functions, provide strong numerical evidence that universal tetramers can arise from the trimer threshold as the four-body scale is moved. These results suggest the possibility of a resonant atom-trimer recombination process near the unitary limit, i.e., when $a_{\mathrm{AT}} \rightarrow \pm \infty$. It is worth noting that the problem of a scale in the tetramer properties has a long 

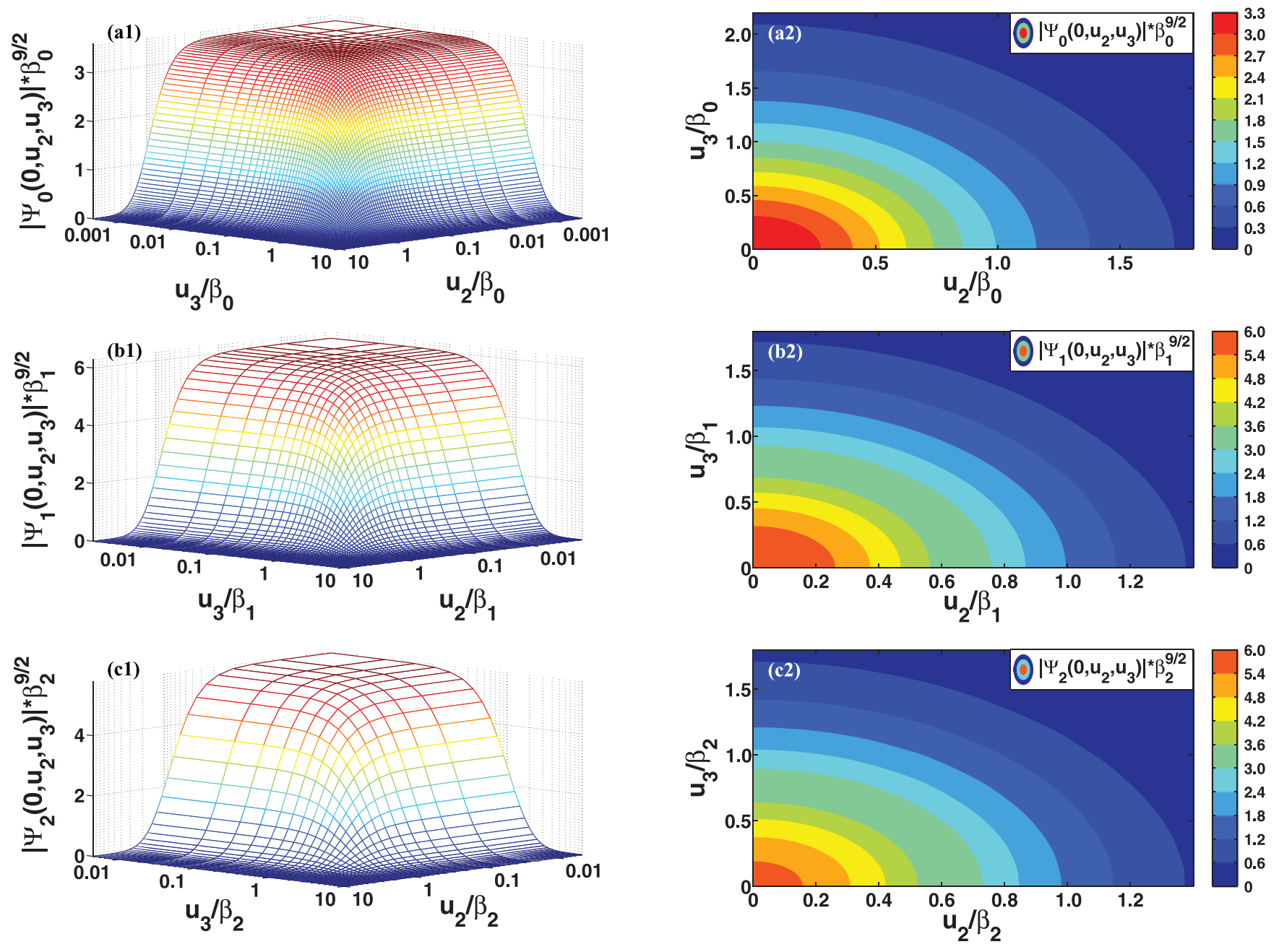

FIG. 12. (Color online) As in Fig. 10, the wave function is given in terms of $u_{2}$ and $u_{3}$, with $u_{1}=0$.

history. The four-body scale gives rise to new scalings of tetramer observables which are not determined only by the two- and three-body properties. We present detailed and accurate numerical results for the binding and structure of tetramer states to support our claim, given conveniently within the framework of scaling functions. As a matter of fact, even results obtained near the unitary limit, by authors arguing that no four-body scale is necessary, are consistent with the universal scaling plot for the tetramer binding energies.

In our numerical results of four-boson bound states, we first consider the exact unitary limit $(1 / a=0)$ and we next consider deviations of this limit to bound $(a>0)$ and virtual $(a<0)$ two-body branches. As shown in Figs. 3-5, universal tetramers can hit the atom-trimer threshold, leading to a resonant relaxation in this channel. This phenomenon could have been, but was not, observed in earlier studies by considering the possibility of a four-body independent scaling behavior near a Feshbach resonance. This could be done by including, for example, tunable three- or four-body potentials, which would allow an independent change of the three- and four-body spectra. The position of the atom-trimer resonance is not only a function of the atom-atom scattering and the three-body scale, but it also depends on the new four-body scale. Our results do not exclude the resonant dimer-dimer recombination
[54] but also add the possibility of a resonant atom-trimer recombination.

Finally, we remark that the four-boson scale can be driven near the Feshbach resonance by induced four-body forces (coming from the one-channel reduction of the atomic interaction) $[15,27]$. Therefore, in this case the Efimov ratio percolating the tetramer observables is not assured anymore. Other universal scaling functions can be derived correlating properties of tetramers, in particular, the one that correlates the binding energies of tetramers attached to different trimers, and as well as by extending our framework to the scattering region. The possibility that tetramers could be formed and driven in cold-atom laboratories, as has been achieved by radio frequency association techniques applied successfully to measure trimer energies [8], will allow experimental verification of the universal scaling relations between tetramer properties, exhibiting a new scale beyond the trimer one.

\section{ACKNOWLEDGMENTS}

We acknowledge partial financial support from the Brazilian agencies Fundação de Amparo à Pesquisa do Estado de São Paulo and Conselho Nacional de Desenvolvimento Científico e Tecnológico. 


\section{APPENDIX A: MOMENTUM-SPACE REPRESENTATION OF FY EQUATIONS}

In the following we present some details of the FY components in the momentum-space representation. By considering the completeness relations Eq. (12) between the permutation operators, we have

$$
\begin{aligned}
\langle u \mid K\rangle= & \int D^{3} u^{\prime}\left\langle u\left|G_{0} t P\right| u^{\prime}\right\rangle\left\langle u^{\prime} \mid K\right\rangle \\
& +\int D^{3} u^{\prime} D^{3} v\left\langle u\left|G_{0} t P\right| u^{\prime}\right\rangle\left\langle u^{\prime} \mid v\right\rangle\langle v \mid H\rangle \\
& +\int D^{3} u^{\prime} D^{3} u^{\prime \prime}\left\langle u\left|G_{0} t P\right| u^{\prime}\right\rangle\left\langle u^{\prime}\left|P_{34}\right| u^{\prime \prime}\right\rangle\left\langle u^{\prime \prime} \mid K\right\rangle \\
\langle v \mid H\rangle= & \int D^{3} v^{\prime}\left\langle v\left|G_{0} t \tilde{P}\right| v^{\prime}\right\rangle\left\langle v^{\prime} \mid H\right\rangle \\
& +\int D^{3} v^{\prime} D^{3} u\left\langle v\left|G_{0} t \tilde{P}\right| v^{\prime}\right\rangle\left\langle v^{\prime} \mid u\right\rangle\langle u \mid K\rangle \\
& +\int D^{3} v^{\prime} D^{3} u D^{3} u^{\prime}\left\langle v\left|G_{0} t \tilde{P}\right| v^{\prime}\right\rangle \\
& \times\left\langle v^{\prime} \mid u\right\rangle\left\langle u\left|P_{34}\right| u^{\prime}\right\rangle\left\langle u^{\prime} \mid K\right\rangle .
\end{aligned}
$$

Therefore to evaluate the above coupled equations (A1), we need to obtain the following matrix elements:

$$
\begin{aligned}
& \left\langle u\left|G_{0} t P\right| u^{\prime}\right\rangle, \quad\left\langle v\left|G_{0} t \tilde{P}\right| v^{\prime}\right\rangle, \\
& \left\langle u\left|P_{34}\right| u^{\prime}\right\rangle, \quad\left\langle u^{\prime} \mid v\right\rangle, \quad\left\langle v^{\prime} \mid u\right\rangle .
\end{aligned}
$$

By considering that in the expressions (A2) we have to insert the two-body matrix elements (14) and that

$$
\begin{array}{r}
\left\langle u|P| u^{\prime}\right\rangle=\int_{-1}^{1} d x \frac{\delta\left[u_{1}-\Pi_{1}\left(u_{2}, u_{2}^{\prime}, x\right)\right]}{u_{1}^{2}} \\
\times \frac{\delta\left[u_{1}^{\prime}-\Pi_{1}\left(u_{2}^{\prime}, u_{2}, x\right)\right]}{u_{1}^{\prime 2}} \frac{\delta\left(u_{3}-u_{3}^{\prime}\right)}{u_{3}^{2}}, \\
\Pi_{1}\left(u_{2}, u_{2}^{\prime}, x\right) \equiv\left|\frac{1}{2} \mathbf{u}_{2}+\mathbf{u}_{2}^{\prime}\right| \\
=\sqrt{\frac{1}{4} u_{2}^{2}+u_{2}^{\prime 2}+u_{2} u_{2}^{\prime 2} x} \\
\left\langle v|\tilde{P}| v^{\prime}\right\rangle=\frac{\delta\left(v_{1}^{\prime}-v_{3}\right)}{v_{1}^{\prime 2}} \frac{\delta\left(v_{3}^{\prime}-v_{1}\right)}{v_{3}^{\prime 2}} \frac{\delta\left(v_{2}^{\prime}-v_{2}\right)}{v_{2}^{\prime 2}},
\end{array}
$$

the matrix elements in (A2) are given by the following:

$$
\begin{aligned}
& \left\langle u\left|G_{0} t P\right| u^{\prime}\right\rangle \\
& =G_{0}\left(u_{1}, u_{2}, u_{3}\right) \int D^{3} u^{\prime \prime}\left\langle u|t| u^{\prime \prime}\right\rangle\left\langle u^{\prime \prime}|P| u^{\prime}\right\rangle \\
& =4 \pi G_{0}\left(u_{1}, u_{2}, u_{3}\right) \chi\left(u_{1}\right) \tau\left(\mathcal{E}_{u}\right) \frac{\delta\left(u_{3}^{\prime}-u_{3}\right)}{u_{3}^{\prime 2}} \\
& \times \int_{-1}^{1} d x \chi\left[\Pi_{1}\left(u_{2}, u_{2}^{\prime}, x\right)\right] \frac{\delta\left[u_{1}^{\prime}-\Pi_{1}\left(u_{2}^{\prime}, u_{2}, x\right)\right]}{u_{1}^{\prime 2}} ; \\
& \left\langle v\left|G_{0} t \tilde{P}\right| v^{\prime}\right\rangle=G_{0}\left(v_{1}, v_{2}, v_{3}\right) \int D^{3} v^{\prime \prime}\left\langle v|t| v^{\prime \prime}\right\rangle\left\langle v^{\prime \prime}|\tilde{P}| v^{\prime}\right\rangle \\
& =4 \pi G_{0}\left(v_{1}, v_{2}, v_{3}\right) \chi\left(v_{1}\right) \chi\left(v_{3}^{\prime}\right) \tau\left(\mathcal{E}_{v}\right) \\
& \quad \times \frac{\delta\left(v_{3}-v_{1}^{\prime}\right)}{v_{3}^{2}} \frac{\delta\left(v_{2}-v_{2}^{\prime}\right)}{v_{2}^{2}} ;
\end{aligned}
$$

$$
\begin{aligned}
\left\langle u\left|P_{34}\right| u^{\prime}\right\rangle= & \frac{\delta\left(u_{1}-u_{1}^{\prime}\right)}{u_{1}^{\prime 2}} \frac{1}{2} \int_{-1}^{1} d x \frac{\delta\left[u_{2}^{\prime}-\Pi_{2}\left(u_{2}, u_{3}, x\right)\right]}{u_{2}^{\prime 2}} \\
& \times \frac{\delta\left[u_{3}^{\prime}-\Pi_{3}\left(u_{2}, u_{3}, x\right)\right]}{u_{3}^{\prime 2}},
\end{aligned}
$$

where

$$
\begin{aligned}
\Pi_{2}\left(u_{2}, u_{3}, x\right) & \equiv\left|\frac{1}{3} \mathbf{u}_{2}+\frac{8}{9} \mathbf{u}_{3}\right| \\
& =\sqrt{\frac{1}{9} u_{2}^{2}+\frac{64}{81} u_{3}^{2}+\frac{16}{27} u_{2} u_{3} x} \\
\Pi_{3}\left(u_{2}, u_{3}, x\right) & \equiv\left|\mathbf{u}_{2}-\frac{1}{3} \mathbf{u}_{3}\right| \\
& =\sqrt{u_{2}^{2}+\frac{1}{9} u_{3}^{2}-\frac{2}{3} u_{2} u_{3} x} ; \\
\left\langle u^{\prime} \mid v\right\rangle=\frac{\delta\left(v_{1}-u_{1}^{\prime}\right)}{v_{1}^{2}} & \frac{1}{2} \int_{-1}^{1} d x \frac{\delta\left[v_{3}-\Pi_{4}\left(u_{2}^{\prime}, u_{3}^{\prime}, x\right)\right]}{v_{3}^{2}} \\
\times \frac{\delta\left[v_{2}-\right.}{\left.\Pi_{5}\left(u_{2}^{\prime}, u_{3}^{\prime}, x\right)\right]} & v_{2}^{2}
\end{aligned}
$$

where

$$
\begin{aligned}
\Pi_{4}\left(u_{2}, u_{3}, x\right) & \equiv\left|\frac{1}{2} \mathbf{u}_{2}+\frac{2}{3} \mathbf{u}_{3}\right| \\
& =\sqrt{\frac{1}{4} u_{2}^{2}+\frac{4}{9} u_{3}^{2}+\frac{2}{3} u_{2} u_{3} x}, \\
\Pi_{5}\left(u_{2}, u_{3}, x\right) & \equiv\left|\mathbf{u}_{2}-\frac{2}{3} \mathbf{u}_{3}\right| \\
& =\sqrt{u_{2}^{2}+\frac{4}{9} u_{3}^{2}-\frac{4}{3} u_{2} u_{3} x}
\end{aligned}
$$

and

$$
\begin{aligned}
\left\langle v^{\prime} \mid u\right\rangle= & \frac{\delta\left(u_{1}-v_{1}^{\prime}\right)}{u_{1}^{2}} \frac{1}{2} \int_{-1}^{1} d x \frac{\delta\left[u_{2}-\Pi_{6}\left(v_{2}^{\prime}, v_{3}^{\prime}, x\right)\right]}{u_{2}^{2}} \\
& \times \frac{\delta\left[u_{3}-\Pi_{7}\left(v_{2}^{\prime}, v_{3}^{\prime}, x\right)\right]}{u_{3}^{2}}
\end{aligned}
$$

where

$$
\begin{aligned}
\Pi_{6}\left(v_{2}, v_{3}, x\right) & \equiv \frac{2}{3}\left|\mathbf{v}_{2}+\mathbf{v}_{3}\right| \\
& =\frac{2}{3} \sqrt{v_{2}^{2}+v_{3}^{2}+2 v_{2} v_{3} x} \\
\Pi_{7}\left(v_{2}, v_{3}, x\right) & \equiv\left|\mathbf{v}_{3}-\frac{1}{2} \mathbf{v}_{2}\right| \\
& =\sqrt{v_{3}^{2}+\frac{1}{4} v_{2}^{2}-v_{2} v_{3} x}
\end{aligned}
$$

\section{APPENDIX B: FOUR-BODY TOTAL WAVE FUNCTIONS IN MOMENTUM SPACE}

The total four-body wave function, which can be written as

$$
\begin{aligned}
|\Psi\rangle= & \left(1+P+P_{34}+P P_{34}+P_{34} P+P P_{34} P\right)|K\rangle \\
& +(1+P+\tilde{P}+P \tilde{P})|H\rangle,
\end{aligned}
$$

is composed of $18 \mathrm{FY}$ components, according to the possible arrangements of the four particles $(i j k l)$. We have 12 of $K$ 
type and 6 of $H$ type, as follows:

$$
\begin{aligned}
& i j k+l \rightarrow\left\{\begin{array}{l}
i j+k+l \equiv K_{i j, k}^{l} \\
j k+i+l \equiv K_{j k, i}^{l}=P_{i j} P_{j k} K_{i j, k}^{l} \\
k i+j+l \equiv K_{k i, j}^{l}=P_{i k} P_{j k} K_{i j, k}^{l}
\end{array}\right. \\
& i j l+k \rightarrow\left\{\begin{array}{l}
i j+l+k \equiv K_{i j, l}^{k}=P_{k l} K_{i j, k}^{l} \\
j l+i+k \equiv K_{j l, i}^{k}=P_{i j} P_{j l} K_{i j, l}^{k} \\
l i+j+k \equiv K_{l i, j}^{k}=P_{i l} P_{j l} K_{i j, l}^{k}
\end{array}\right. \\
& i k l+j \rightarrow\left\{\begin{array}{l}
i k+l+j \equiv K_{i k, l}^{j}=P_{j k} P_{k l} K_{i j, k}^{l} \\
l i+k+j \equiv K_{l i, k}^{j}=P_{i l} P_{l k} K_{i k, l}^{j} \\
k l+i+j \equiv K_{k l, i}^{j}=P_{i k} P_{l k} K_{i k, l}^{j}
\end{array}\right. \\
& i k l+i \rightarrow\left\{\begin{array}{l}
j k+l+i \equiv K_{j k, l}^{i}=P_{i j} P_{j k} P_{k l} K_{i j, k}^{l} \\
l j+k+i \equiv K_{l j, k}^{i}=P_{l k} P_{k j} K_{j k, l}^{i} \\
k l+j+i \equiv K_{k l, j}^{i}=P_{l j} P_{k j} K_{j k, l}^{i}
\end{array}\right. \\
& i k+j l \rightarrow\left\{\begin{array}{l}
i k+j+l \equiv H_{i k, j l}=P_{j k} H_{i j, k l} \\
j l+i+k \equiv H_{j l, i k}=P_{j k} P_{i k} P_{j l} H_{i j, k l}
\end{array}\right. \\
& i\left\{\begin{array}{l}
i j+k+l \equiv H_{i j, k l} \\
k l+i+j \equiv H_{k l, i j}=P_{i k} P_{j l} H_{i j, k l} \\
j k+i+l \equiv H_{j k, i l}=P_{i j} P_{j k} H_{i j, k l} .
\end{array}\right.
\end{aligned}
$$

In momentum space, we have

$$
\begin{aligned}
\langle u \mid \Psi\rangle= & \langle u|1| K\rangle+\langle u|P| K\rangle+\left\langle u\left|P_{34}\right| K\right\rangle \\
& +\left\langle u\left|P P_{34}\right| K\right\rangle+\left\langle u\left|P_{34} P\right| K\right\rangle+\left\langle u\left|P P_{34} P\right| K\right\rangle \\
& +\langle u|1| H\rangle+\langle u|P| H\rangle+\langle u|\tilde{P}| H\rangle+\langle u|P \tilde{P}| H\rangle \\
\equiv & \sum\left[K_{\mathcal{O}}+H_{\mathcal{O}}\right] ; \quad \mathcal{O}=1, P, P_{34}, P P_{34}, \ldots
\end{aligned}
$$

where

$$
\begin{aligned}
K_{\mathcal{O}} & \equiv\langle u|\mathcal{O}| K\rangle=\int D^{3} u^{\prime}\left\langle u|\mathcal{O}| u^{\prime}\right\rangle\left\langle u^{\prime} \mid K\right\rangle, \\
& =\int D^{3} u^{\prime}\left\langle u|\mathcal{O}| u^{\prime}\right\rangle G_{0}\left(u_{1}^{\prime}, u_{2}^{\prime}, u_{3}^{\prime}\right) \chi\left(u_{1}^{\prime}\right) \mathcal{K}\left(u_{2}^{\prime}, u_{3}^{\prime}\right),
\end{aligned}
$$

and

$$
\begin{aligned}
H_{\mathcal{O}} & \equiv\langle u|\mathcal{O}| H\rangle=\int D^{3} v^{\prime}\left\langle u|\mathcal{O}| v^{\prime}\right\rangle\left\langle v^{\prime} \mid H\right\rangle \\
& =\int D^{3} v^{\prime}\left\langle u|\mathcal{O}| v^{\prime}\right\rangle G_{0}\left(v_{1}^{\prime}, v_{2}^{\prime}, v_{3}^{\prime}\right) \chi\left(v_{1}^{\prime}\right) \mathcal{H}\left(v_{2}^{\prime}, v_{3}^{\prime}\right) .
\end{aligned}
$$

In the following we present the matrix elements of $K_{\mathcal{O}}$ and $H_{\mathcal{O}}$ explicitly:

$$
\begin{aligned}
K_{1} \equiv\langle u|1| K\rangle & \\
= & K\left(u_{1}, u_{2}, u_{3}\right)=G_{0}\left(u_{1}, u_{2}, u_{3}\right) \mathcal{K}\left(u_{2}, u_{3}\right), \\
K_{P} & \equiv\langle u|P| K\rangle=\int d x_{12} K\left(\Pi_{P}^{1}, \Pi_{P}^{2}, u_{3}\right) \\
& =\int d x_{12} G_{0}\left(\Pi_{P}^{1}, \Pi_{P}^{2}, u_{3}\right) \mathcal{K}\left(\Pi_{P}^{2}, u_{3}\right),
\end{aligned}
$$

where

$$
\begin{gathered}
\Pi_{P}^{1} \equiv \Pi_{P}^{1}\left(u_{1}, u_{2}, x_{12}\right)=\left|-\frac{1}{2} \mathbf{u}_{1}-\frac{3}{4} \mathbf{u}_{2}\right| \\
=\sqrt{\frac{1}{4} u_{1}^{2}+\frac{9}{16} u_{2}^{2}+\frac{3}{4} u_{1} u_{2} x_{12},} \\
\Pi_{P}^{2} \equiv \Pi_{P}^{2}\left(u_{1}, u_{2}, x_{12}\right)=\left|\mathbf{u}_{1}-\frac{1}{2} \mathbf{u}_{2}\right| \\
=\sqrt{u_{1}^{2}+\frac{1}{4} u_{2}^{2}-u_{1} u_{2} x_{12}} ; \\
K_{P_{34}} \equiv\left\langle u\left|P_{34}\right| K\right\rangle=\frac{1}{2} \int d x_{23} K\left(u_{1}, \Pi_{P_{34}}^{2}, \Pi_{P_{34}}^{3}\right) \\
=\frac{1}{2} \int d x_{23} G_{0}\left(u_{1}, \Pi_{P_{34}}^{2}, \Pi_{P_{34}}^{3}\right) \mathcal{K}\left(\Pi_{P_{34}}^{2}, \Pi_{P_{34}}^{3}\right),
\end{gathered}
$$

where

$$
\begin{aligned}
\Pi_{P_{34}}^{2} & \equiv \Pi_{P_{34}}^{2}\left(u_{2}, u_{3}, x_{23}\right)=\left|\frac{1}{3} \mathbf{u}_{2}+\frac{8}{9} \mathbf{u}_{3}\right| \\
& =\sqrt{\frac{1}{9} u_{2}^{2}+\frac{64}{81} u_{3}^{2}+\frac{16}{27} u_{2} u_{3} x_{23},} \\
\Pi_{P_{34}}^{3} & \equiv \Pi_{P_{34}}^{3}\left(u_{2}, u_{3}, x_{23}\right)=\left|\mathbf{u}_{2}-\frac{1}{3} \mathbf{u}_{3}\right| \\
& =\sqrt{u_{2}^{2}+\frac{1}{9} u_{3}^{2}-\frac{2}{3} u_{2} u_{3} x_{23}} ;
\end{aligned}
$$

$$
\begin{aligned}
K_{P P_{34}} \equiv & \left\langle u\left|P P_{34}\right| K\right\rangle \\
= & \frac{1}{2} \int d x_{12} \int d x_{12,3} K\left(\Pi_{P P_{34}}^{1}, \Pi_{P P_{34}}^{2}, \Pi_{P P_{34}}^{3}\right) \\
= & \frac{1}{2} \int d x_{12} \int d x_{12,3} G_{0}\left(\Pi_{P P_{34}}^{1}, \Pi_{P P_{34}}^{2}, \Pi_{P P_{34}}^{3}\right) \\
& \times \mathcal{K}\left(\Pi_{P P_{34}}^{2}, \Pi_{P P_{34}}^{3}\right)
\end{aligned}
$$

where

$$
\begin{gathered}
\Pi_{P P_{34}}^{1}=\Pi_{P}^{1}\left(u_{1}, u_{2}, x_{12}\right), \\
\Pi_{P P_{34}}^{2}=\Pi_{P_{34}}^{2}\left(\Pi_{P}^{2}\left(u_{1}, u_{2}, x_{12}\right), u_{3}, x_{12,3}\right), \\
\Pi_{P P_{34}}^{3}=\Pi_{P_{34}}^{3}\left(\Pi_{P}^{2}\left(u_{1}, u_{2}, x_{12}\right), u_{3}, x_{12,3}\right) ;
\end{gathered}
$$

$$
\begin{aligned}
K_{P_{34} P} \equiv & \left\langle u\left|P_{34} P\right| K\right\rangle \\
= & \frac{1}{2} \int d x_{23} \int d x_{23,1} K\left(\Pi_{P_{34} P}^{1}, \Pi_{P_{34} P}^{2}, \Pi_{P_{34} P}^{3}\right) \\
= & \frac{1}{2} \int d x_{23} \int d x_{23,1} G_{0}\left(\Pi_{P_{34} P}^{1}, \Pi_{P_{34} P}^{2}, \Pi_{P_{34} P}^{3}\right) \\
& \times \mathcal{K}\left(\Pi_{P_{34} P}^{2}, \Pi_{P_{34} P}^{3}\right),
\end{aligned}
$$

where

$$
\begin{aligned}
& \Pi_{P_{34} P}^{1}=\Pi_{P}^{1}\left(u_{1}, \Pi_{P_{34}}^{2}\left(u_{2}, u_{3}, x_{23}\right), x_{23,1}\right), \\
& \Pi_{P_{34} P}^{2}=\Pi_{P}^{2}\left(\left(u_{1}, \Pi_{P_{34}}^{2}\left(u_{2}, u_{3}, x_{23}\right), x_{23,1}\right),\right. \\
& \Pi_{P_{34} P}^{3}=\Pi_{P_{34}}^{3}\left(u_{2}, u_{3}, x_{23}\right)
\end{aligned}
$$




$$
\begin{aligned}
K_{P P_{34} P} & \equiv\left\langle u\left|P P_{34} P\right| K\right\rangle=\frac{1}{2} \int d x_{12} \int d x_{12,3} \int d x_{12 ; 12,3} K\left(\Pi_{P P_{34} P}^{1}, \Pi_{P P_{34} P}^{2}, \Pi_{P P_{34} P}^{3}\right) \\
& =\frac{1}{2} \int d x_{12} \int d x_{12,3} \int d x_{12 ; 12,3} G_{0}\left(\Pi_{P P_{34} P}^{1}, \Pi_{P P_{34} P}^{2}, \Pi_{P P_{34} P}^{3}\right) \mathcal{K}\left(\Pi_{P P_{34} P}^{2}, \Pi_{P P_{34} P}^{3}\right),
\end{aligned}
$$

where

$$
\begin{gathered}
\Pi_{P P_{34} P}^{1}=\Pi_{P}^{1}\left(\Pi_{P}^{1}\left(u_{1}, u_{2}, x_{12}\right), \Pi_{P_{34}}^{2}\left[\Pi_{P}^{2}\left(u_{1}, u_{2}, x_{12}\right), u_{3}, x_{12,3}\right], x_{12 ; 12,3}\right), \\
\Pi_{P P_{34} P}^{2}=\Pi_{P}^{2}\left(\Pi_{P}^{1}\left(u_{1}, u_{2}, x_{12}\right), \Pi_{P_{34}}^{2}\left[\Pi_{P}^{2}\left(u_{1}, u_{2}, x_{12}\right), u_{3}, x_{12,3}\right], x_{12 ; 12,3}\right), \\
\Pi_{P P_{34} P}^{3}=\Pi_{P_{34}}^{3}\left(\Pi_{P}^{2}\left(u_{1}, u_{2}, x_{12}\right), u_{3}, x_{12,3}\right) .
\end{gathered}
$$

For the matrix elements of $H_{\mathcal{O}}$, we also need to evaluate the transformation between the two different representations, i.e., the $K$ and $H$ type,

$$
\begin{aligned}
H_{1} & \equiv\langle u|1| H\rangle=\frac{1}{2} \int d x_{23} H\left(u_{1}, \Sigma_{1}^{2}, \Sigma_{1}^{3}\right) \\
& =\frac{1}{2} \int d x_{23,1} G_{0}\left(u_{1}, \Sigma_{1}^{2}, \Sigma_{1}^{3}\right) \mathcal{H}\left(\Sigma_{1}^{2}, \Sigma_{1}^{3}\right),
\end{aligned}
$$

where

$$
\begin{aligned}
\Sigma_{1}^{2} & \equiv \Sigma_{1}^{2}\left(u_{2}, u_{3}, x_{23}\right)=\left|\mathbf{u}_{2}+\frac{2}{3} \mathbf{u}_{3}\right| \\
& =\sqrt{u_{2}^{2}+\frac{4}{9} u_{3}^{2}+\frac{4}{3} u_{2} u_{3} x_{23}}, \\
\Sigma_{1}^{3} & \equiv \Sigma_{1}^{3}\left(u_{2}, u_{3}, x_{23}\right)=\left|\frac{1}{2} \mathbf{u}_{2}-\frac{2}{3} \mathbf{u}_{3}\right| \\
& =\sqrt{\frac{1}{4} u_{2}^{2}+\frac{4}{9} u_{3}^{2}-\frac{2}{3} u_{2} u_{3} x_{23}} ;
\end{aligned}
$$

$$
\begin{aligned}
H_{P} & \equiv\langle u|P| H\rangle \\
& =\frac{1}{2} \int d x_{12} \int d x_{12,3} H\left(\Sigma_{P}^{1}, \Sigma_{P}^{2}, \Sigma_{P}^{3}\right) \\
& =\frac{1}{2} \int d x_{12} \int d x_{12,3} G_{0}\left(\Sigma_{P}^{1}, \Sigma_{P}^{2}, \Sigma_{P}^{3}\right) \mathcal{H}\left(\Sigma_{P}^{2}, \Sigma_{P}^{3}\right),
\end{aligned}
$$

where

$$
\begin{gathered}
\Sigma_{P}^{1}=\Pi_{P}^{1}\left(u_{1}, u_{2}, x_{12}\right), \\
\Sigma_{P}^{2}=\Sigma_{1}^{2}\left(\Pi_{P}^{2}\left(u_{1}, u_{2}, x_{12}\right), u_{3}, x_{12,3}\right), \\
\Sigma_{P}^{3}=\Sigma_{1}^{3}\left(\Pi_{P}^{2}\left(u_{1}, u_{2}, x_{12}\right), u_{3}, x_{12,3}\right) ; \\
H_{\tilde{P}} \equiv\langle u|\tilde{P}| H\rangle=\frac{1}{2} \int d x_{23} H\left(\Sigma_{\tilde{P}}^{1}, \Sigma_{\tilde{P}}^{2}, u_{1}\right) \\
=\frac{1}{2} \int d x_{23} G_{0}\left(\Sigma_{\tilde{P}}^{1}, \Sigma_{\tilde{P}}^{2}, u_{1}\right) \mathcal{H}\left(\Sigma_{\tilde{P}}^{2}, u_{1}\right),
\end{gathered}
$$

where

$$
\begin{gathered}
\Sigma_{\tilde{P}}^{1}=\Sigma_{1}^{3}\left(u_{2}, u_{3}, x_{23}\right), \\
\Sigma_{\tilde{P}}^{2}=\Sigma_{1}^{2}\left(u_{2}, u_{3}, x_{23}\right) ; \\
H_{P \tilde{P}} \equiv\langle u|P \tilde{P}| H\rangle \\
=\frac{1}{2} \int d x_{12} \int d x_{12,3} H\left(\Sigma_{P \tilde{P}}^{1}, \Sigma_{P \tilde{P}}^{2}, \Sigma_{P \tilde{P}}^{3}\right)
\end{gathered}
$$

$$
\begin{aligned}
= & \frac{1}{2} \int d x_{12} \int d x_{12,3} G_{0}\left(\Sigma_{P \tilde{P}}^{1}, \Sigma_{P \tilde{P}}^{2}, \Sigma_{P \tilde{P}}^{3}\right) \\
& \times \mathcal{H}\left(\Sigma_{P \tilde{P}}^{2}, \Sigma_{P \tilde{P}}^{3}\right),
\end{aligned}
$$

where

$$
\begin{gathered}
\Sigma_{P \tilde{P}}^{1}=\Sigma_{1}^{3}\left(\Pi_{P}^{2}\left(u_{1}, u_{2}, x_{12}\right), u_{3}, x_{12,3}\right), \\
\Sigma_{P \tilde{P}}^{2}=\Sigma_{1}^{2}\left(\Pi_{P}^{2}\left(u_{1}, u_{2}, x_{12}\right), u_{3}, x_{12,3}\right), \\
\Sigma_{P \tilde{P}}^{3}=\Pi_{P}^{1}\left(u_{1}, u_{2}, x_{12}\right) .
\end{gathered}
$$

\section{APPENDIX C: ON THE NUMERICAL APPROACH}

The final set of homogeneous coupled integral equations in momentum space, after discretization, defines a huge matrix eigenvalue equation, which is solved by iteration within a Lanczos-like method. This method is quite efficient in solving few-body systems. For continuous momentum and angle variable discretization we have used Gaussian-quadrature grid points with hyperbolic and linear mappings, respectively. After considering a convenient mesh distribution to have optimal numerical stability, the number of grid points found necessary in mapping the momentum variable was set up to 140 . That was achieved by studying the concentration of the points in the different relevant regions of momentum integration. For more details on the general numerical techniques that we are considering, see also Ref. [55].

Next, we also address problems concerning the accuracy and precision of the results in the unitary limit.

\section{Numerical convergence}

The numerical convergence of our results is exemplified by one case where stability is more difficult to achieve. As this happens in the excited states of large scale ratios, we choose the case of $\mu_{4} / \mu_{3}=300$, in which we can verify the existence of up to four tetramers. The number of points and, particularly, their distribution, is quite critical for the accuracy of the excited-state energies and corresponding FY reduced amplitudes. In our systematic study to construct the limit cycle shown in Fig. 5, we are satisfied to achieve $\sim 1 \%$ of deviation from the converged results. To be clear, we choose to present in Fig. 13 the convergence with respect to the mesh number of points for the first and second excited states.

As the corresponding FY components become quite concentrated at the momentum origin, in this case the momentumspace discretization $u_{i}$ is derived from the quadrature Gaussian 

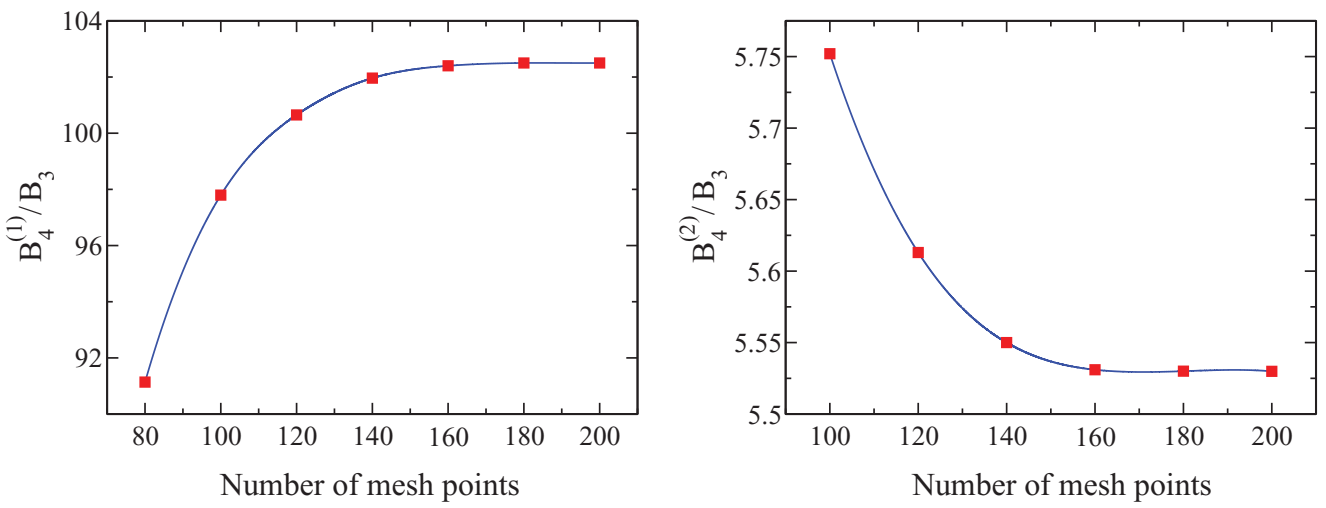

FIG. 13. (Color online) Convergence of numerical results for the first- and second-excited-state energies, in the case that $\mu_{4} / \mu_{3}=300$.

mesh distribution $x_{i} \equiv\{-1,+1\}$ by the following hyperbolic mapping:

$$
u_{i}=\frac{1+x_{i}}{c_{1}\left(1-x_{i}\right)+c_{2} x_{i}},
$$

with $c_{1} \equiv \mu_{4} / \mu_{3}$ and $c_{2}=0.4$. In general, we observe that for all the cases with smaller scaling ratios, or for excited states that are not too close to the continuum threshold, i.e., about to be unbound, 140 points are enough to achieve less than $1 \%$ of inaccuracy, within an appropriate mesh distribution.

\section{Iterative Lanczos-type diagonalization algorithm for solution of the coupled FY equations}

The coupled FY integral equations (24) can be schematically represented as an eigenvalue problem:

$$
\mathbb{k}(E) \cdot \psi=\lambda(E) \psi,
$$

where the kernel of integral equations $\mathbb{k}(E)$ is energy dependent, and $\lambda(E)$ and $\psi$ are its eigenvalue and eigenvector, correspondingly. The vector $\psi$ is composed of FY components as $\psi=\left({ }_{\mathcal{H}}^{\mathcal{K}}\right)$. The binding energy of the four-body bound state can be obtained when one of the eigenvalues obtained from the solution of Eq. (C2) for an input energy becomes one $[\lambda(E)=1]$. To this aim one should solve the eigenvalue equation $(\mathrm{C} 2)$ for a set of input energies.

After discretization of continuous momentum and angle variables, the kernel of the eigenvalue equation turns into a huge matrix with dimensionality of $120 \times 120 \times 120 \times 40 \times$ $40 \sim 10^{9}$, where we have used 120 mesh points for Jacobi momentum variables (with a hyperbolic mapping) and 40 mesh points for angle variables (with a linear mapping). Considering that exact solutions of huge matrices by diagonalization are not so efficient, we avoid such procedures by using a Lanczos-type technique, which is based on iteration. In this way one can obtain the eigenvalues and eigenvectors of a huge matrix, from input energies [56]. In the following, we describe some details of this technique.

The iteration procedure is performed with a properly starting vector $\psi_{0}=\left(\begin{array}{c}\mathcal{K}_{0} \\ \mathcal{H}_{0}\end{array}\right)$, chosen as Gaussian functions for both $\mathcal{K}$ and $\mathcal{H}$ components. After $N$ iterations, one obtains the set of vectors $\left\{\psi_{i}\right\}=\psi_{1}, \psi_{2}, \ldots, \psi_{N}$ where

$$
\psi_{i}=\mathbb{k}(E) \cdot \psi_{i-1} ; \quad i=1,2, \ldots, N .
$$

In the iterative diagonalization approach, an orthonormal basis $\left\{\bar{\psi}_{i}\right\}$ can be built up by following a recursive procedure and by using the original vector set $\left\{\psi_{i}\right\}$, where

$$
\bar{\psi}_{i}=c_{i}\left\{\psi_{i}-\sum_{j=1}^{i-1}\left(\bar{\psi}_{j} \cdot \psi_{i}\right) \bar{\psi}_{j}\right\}(i=1,2, \ldots, N) .
$$

In the above, $c_{i}$ are normalization factors which can be obtained by orthogonalization of orthonormal basis states $\bar{\psi}_{i} \cdot \bar{\psi}_{j}=\delta_{i j}$. By introducing a matrix $D$, obtained from the FY components as

$$
\begin{aligned}
D_{i j}=\psi_{i} \cdot \psi_{j} & =\left(\mathcal{K}_{i} \mathcal{H}_{i}\right) \cdot\left(\begin{array}{c}
\mathcal{K}_{j} \\
\mathcal{H}_{j}
\end{array}\right) \\
& =\mathcal{K}_{i} \cdot \mathcal{K}_{j}+\mathcal{H}_{i} \cdot \mathcal{H}_{j}=D_{i j}^{K}+D_{i j}^{H},
\end{aligned}
$$

where

$$
\begin{aligned}
& D_{i j}^{K}=\mathcal{K}_{i} \cdot \mathcal{K}_{j}=\int d u_{2} u_{2}^{2} \int d u_{3} u_{3}^{2} \mathcal{K}_{i}\left(u_{2}, u_{3}\right) \mathcal{K}_{j}\left(u_{2}, u_{3}\right), \\
& D_{i j}^{H}=\mathcal{H}_{i} \cdot \mathcal{H}_{j}=\int d v_{2} v_{2}^{2} \int d v_{3} v_{3}^{2} \mathcal{H}_{i}\left(v_{2}, v_{3}\right) \mathcal{H}_{j}\left(v_{2}, v_{3}\right),
\end{aligned}
$$

the normalization factors $c_{i}$ can be obtained as

$$
c_{i}=\frac{1}{\sqrt{D_{i i}-\sum_{j=1}^{i-1} N_{i j}^{2}}} .
$$

The matrix elements $N_{i j}$ are a projection of vectors $\psi_{i}$ on $\bar{\psi}_{j}$, i.e., $N_{i j}=\psi_{i} \cdot \bar{\psi}_{j}$,

$$
N_{i j}=c_{j}\left\{D_{i j}-\sum_{k=1}^{j-1} N_{i k} \cdot N_{j k}\right\} .
$$

By expanding the original vector $\psi$ in Eq. (C2), in terms of constructed orthonormal vectors,

$$
\psi=\sum_{i=1}^{N-1} g_{i} \bar{\psi}_{i}
$$

and projecting the eigenvalue equation onto $\bar{\psi}_{j}$, the huge dimension of the eigenvalue problem is reduced to a problem 
of much smaller dimension $N-1$ :

$$
\sum_{i=1}^{N-1} M_{i j} g_{i}=\lambda g_{j}
$$

where

$$
M_{i j} \equiv \bar{\psi}_{i} \cdot \mathbb{k} \cdot \bar{\psi}_{j}
$$

By defining $a_{i j}$ and $b_{i j}$ as coefficients of linear expansion of original vectors in terms of orthonormal vectors and reversely,

$$
\psi_{i}=\sum_{j=1}^{i} a_{i j} \bar{\psi}_{j}, \quad \bar{\psi}_{i}=\sum_{j=1}^{i} b_{i j} \psi_{j},
$$

the matrix elements of $M$ can be obtained as

$$
M_{i j}=\sum_{k=1}^{i} b_{i k} a_{k+1 j}, \quad 1 \leqslant i, j<N,
$$

where

$$
a_{i j}= \begin{cases}N_{i j} & j<i \\ \frac{1}{c_{i}} & j=i\end{cases}
$$

$$
b_{i j}= \begin{cases}c_{j}\left(-\sum_{k=j+1}^{i} b_{i k} a_{k j}\right) & j<i \\ \frac{1}{c_{i}} & j=i .\end{cases}
$$

The reduced eigenvalue problem, Eq. (C10), can be solved easily by diagonalization of the matrix $M$. For a given input energy, one can obtain a set of eigenvalues and their corresponding eigenvectors. In order to obtain the physical binding energy, one should look for an eigenvalue $\lambda=1$ in the obtained eigenvalue spectrum. Clearly, the eigenvector corresponding to this eigenvalue gives the coefficients $g_{i}$. Therefore, by using the Eq. (C9), one can obtain the physical eigenvector or the FY components.
[1] K. G. Wilson, Phys. Rev. D 3, 1818 (1971).

[2] T. Frederico, L. Tomio, A. Delfino, and A. E. A. Amorim, Phys. Rev. A 60, R9 (1999).

[3] P. F. Bedaque, H.-W. Hammer, and U. van Kolck, Phys. Rev. Lett. 82, 463 (1999).

[4] R. F. Mohr, R. J. Furnstahl, H.-W. Hammer, R. J. Perry, and K. G. Wilson, Ann. Phys. 321, 225 (2006).

[5] V. Efimov, Phys. Lett. B 33, 563 (1970); Sov. J. Nucl. Phys. 12, 589 (1971).

[6] T. Kraemer, M. Mark, P. Waldburger, J. G. Danzl, C. Chin, B. Engeser, A. D. Lange, K. Pilch, A. Jaakkola, H.-C. Nägerl, and R. Grimm, Nature (London) 440, 315 (2006).

[7] G. Barontini, C. Weber, F. Rabatti, J. Catani, G. Thalhammer, M. Inguscio, and F. Minardi, Phys. Rev. Lett. 103, 043201 (2009).

[8] T. Lompe, T. B. Ottenstein, F. Serwane, A. N. Wenz, G. Zürn, and S. Jochim, Science 330, 940 (2010).

[9] F. Ferlaino and R. Grimm, Physics 3, 9 (2010).

[10] R. D. Amado and F. C. Greenwood, Phys. Rev. D 7, 2517 (1973).

[11] H. Kröger and R. Perne, Phys. Rev. C 22, 21 (1980).

[12] S. K. Adhikari and A. C. Fonseca, Phys. Rev. D 24, 416 (1981).

[13] H. W. L. Naus and J. A. Tjon, Few-Body Syst. 2, 121 (1987).

[14] L. Platter, H. W. Hammer, and Ulf-G. Meissner, Phys. Rev. A 70, 052101 (2004).

[15] M. T. Yamashita, L. Tomio, A. Delfino, and T. Frederico, Europhys. Lett. 75, 555 (2006).

[16] H.-W. Hammer and L. Platter, Eur. Phys. J. A 32, 113 (2007).

[17] J. von Stecher, J. P. D'Incao, and C. H. Greene, Nat. Phys. 5, 417 (2009).

[18] Y. Wang and B. D. Esry, Phys. Rev. Lett. 102, 133201 (2009).

[19] A. Deltuva, Phys. Rev. A 82, 040701(R) (2010), e-print arXiv:1009.1295v1 [physics.atm-clus].

[20] J. von Stecher, J. Phys. B 43, 101002 (2010).

[21] R. Lazauskas and J. Carbonell, Phys. Rev. A 73, 062717 (2006).

[22] F. Ferlaino, S. Knoop, M. Berninger, W. Harm, J. P. Dincao, H. C. Hagerl, and R. Grimm, Phys. Rev. Lett. 102, 140401 (2009).

[23] M. Zaccanti, B. Deissler, C. D'Errico, M. Fattori, M. JonaLasinio, S. Müller, G. Roati, M. Inguscio, and G. Modugno, Nat. Phys. 5, 586 (2009).
[24] S. E. Pollack, D. Dries, and R. G. Hulet, Science 326, 1683 (2009), and www.sciencemag.org/cgi/content/full/1182840/ DC1 for supporting online material.

[25] G. Modugno, Science 326, 1640 (2009).

[26] M. R. Hadizadeh, M. T. Yamashita, L. Tomio, A. Delfino, and T. Frederico, Phys. Rev. Lett. 107, 135304 (2011).

[27] S. Nakajima, M. Horikoshi, T. Mukaiyama, P. Naidon, and M. Ueda, Phys. Rev. Lett. 105, 023201 (2010); S. Nakajima, M. Horikoshi, T. Mukaiyama, P. Naidon, and M. Ueda, ibid. 106, 143201 (2011).

[28] L. D. Faddeev, Zh. Eksp. Teor. Fiz. 39, 1459 (1960) [Sov. Phys. JETP 12, 1014 (1961)].

[29] O. A. Yakubovsky, Yad. Fiz. 5, 1312 (1967) [Sov. J. Nucl. Phys. 5, 937 (1967)].

[30] S. Weinberg, Phys. Rev. 131, 440 (1963).

[31] J. A. Tjon, Phys. Lett. B 56, 217 (1975).

[32] T. Sasakawa, Phys. Rev. C 13, 1801 (1976).

[33] B. F. Gibson and D. R. Lehman, Phys. Rev. C 14, 685 (1976); B. F. Gibson and D. R. Lehman, ibid. 15, 2257 (1977).

[34] S. P. Merkuriev, S. L. Yakovlev, and C. Gignoux, Nucl. Phys. A 431, 125 (1984).

[35] N. W. Schellingerhout, J. J. Schut, and L. P. Kok, Phys. Rev. C 46, 1192 (1992).

[36] H. Kamada and W. Glöckle, Phys. Lett. B 292, 1 (1992); H. Kamada et al., Phys. Rev. C 64, 044001 (2001).

[37] A. L. Zubarev and V. B. Mandelzweig, Phys. Rev. C 52, 509 (1995).

[38] I. N. Filikhin, S. L. Yakovlev, V. A. Roudnev, and B. Vlahovic, J. Phys. A: At. Mol. Opt. Phys. 35, 501 (2002).

[39] R. Lazauskas and J. Carbonell, Few-Body Syst. 34, 105 (2004).

[40] S. Bayegan, M. R. Hadizadeh, and W. Glöckle, Prog. Theor. Phys. 120, 887 (2008).

[41] M. R. Hadizadeh, L. Tomio, and S. Bayegan, Phys. Rev. C 83, 054004 (2011).

[42] R. Perne and W. Sandhas, Phys. Rev. Lett. 39, 788 (1977).

[43] S. A. Sofianos, H. Fiedeldey, H. Haberzettl, and W. Sandhas, Phys. Rev. C 26, 228 (1982).

[44] S. Nakaichi-Maeda and T. K. Lim, Phys. Rev. A 28, 692 (1983).

[45] A. C. Fonseca, Phys. Rev. C 30, 35 (1984). 
[46] E. O. Alt, P. Grassberger, and W. Sandhas, Phys. Rev. C 1, 85 (1970).

[47] L. H. Thomas, Phys. Rev. 47, 903 (1935).

[48] S. K. Adhikari, A. Delfino, T. Frederico, I. D. Goldman, and L. Tomio, Phys. Rev. A 37, 3666 (1988).

[49] L. Tomio, M. T. Yamashita, T. Frederico, and F. Bringas, Laser Phys. 21, 1464 (2011).

[50] M. Thøgersen, D. V. Fedorov, and A. S. Jensen, Phys. Rev. A 78, 020501(R) (2008).

[51] Th. Cornelius and W. Glöckle, J. Chem. Phys. 85, 1 (1986).
[52] D. Blume and C. Greene, J. Chem. Phys. 112, 8053 (2000).

[53] L. Platter, C. Ji, and D. R. Phillips, Phys. Rev. A 79, 022702 (2009).

[54] J. P. D'Incao, J. von Stecher, and C. H. Greene, Phys. Rev. Lett. 103, 033004 (2009).

[55] M. R. Hadizadeh and S. Bayegan, Few-Body Syst. 40, 171 (2007).

[56] A. Stadler, W. Glöckle, and P. U. Sauer, Phys. Rev. C 44, 2319 (1991).

[57] T. Frederico, L. Tomio, A. Delfino, M. R. Hadizadeh, and M. T. Yamashita, Few-Body Syst. 51, 87 (2011). 